\title{
REDUCED MODELS FOR STRUCTURES IN THE MEDIUM-FREQUENCY RANGE COUPLED WITH INTERNAL ACOUSTIC CAVITIES
}

\author{
Christian Soize
}

Structural Dynamics and Coupled Systems Department, ONERA, BP 72, F-92322 Chatillon Cedex, France

\begin{abstract}
This paper concerns the development of a method adapted for constructing reduced models in the medium-frequency range to a general three-dimensional dissipative structure consisting of an anisotropic, inhomogeneous, viscoelastic bounded medium coupled with an internal acoustic cavity. The reduced models are obtained using the Ritz-Galerkin method for which the projection subspace corresponds to the dominant eigensubspace of the energy operator of the structure in the medium-frequency band of analysis. Two fundamental cases are considered: 1) both the structure and the internal acoustic cavity have a medium-frequency behavior in the frequency band of analysis; 2) the structure has a medium-frequency behavior in the frequency band of analysis while the internal acoustic cavity has a low-frequency behavior.
\end{abstract}

PACS numbers: 43.40

\section{INTRODUCTION}

This paper is the continuation of two previous papers published by the author, concerning the development of a method for constructing reduced models in the medium-frequency (MF) range for general structural dynamics systems ${ }^{1}$ (structures in a vacuum) and external structural-acoustic systems ${ }^{2}$ (structure coupled with an external acoustic fluid (gas or liquid)).

For a mechanical system such as a structure in a vacuum or a structural-acoustic system, its response in the frequency domain (its "behavior") depends on the frequency range defined as follows (see Ref. 3).

The low-frequency range (LF) can be defined as the modal domain for which the associated conservative system has a small number of modes; there are no modal overlaps due to the dissipation effects. In this LF range, the finite element method can be used for spatial discretization and the dynamic analysis can be performed in the frequency domain using reduced models which are very efficient and popular tools in constructing the solution. Such reduced models correspond to a Ritz-Galerkin reduction of the dynamical model using the normal modes corresponding to the lowest eigenfrequencies of the associated conservative system. The efficiency of this kind of reduced model is due to the small number of generalized dynamical degrees of freedom used in the representation and in addition, is obtained by solving a well-stated generalized symmetric eigenvalue problem for which only the first eigenvalues and the corresponding eigenfunctions have to be calculated. In addition when such a reduced model is obtained, responses to any deterministic or random excitations can be calculated for no significant additional numerical cost.

The high-frequency range (HF) can be defined as the frequency band for which there is a uniform high modal density; there is a uniform modal overlap due to the high modal density 
and the dissipation effects. For this HF range, the finite element method cannot be used for spatial discretization and the dynamic analysis has to be performed using the wave approach, the global statistical energy approach (such as the very popular Statistical Energy Analysis) and the local energy approach (such as the power flow analysis based on continuous energy equations).

For complex systems such as general three-dimensional structures, an intermediate frequency range called medium-frequency range (MF) appears. The modal density exhibits large variations over the band. In addition, if there is a structural complexity related to the presence of fuzzy substructures, mechanical models have to be adapted. This MF range cannot be analyzed with the tools used for the LF and HF ranges. A complex structure is then considered as a master structure coupled with all the dynamical subsystems (fuzzy substructures). In the MF range, probabilistic models have to be used to model the effects of fuzzy substructures on the master structure and the finite element method can only be used for spatial discretization of the master structure. In addition, random uncertainties have to be modeled in the master structure in order to increase robustness of response predictions. Concerning dynamical analysis of the master structure, the modal method which is very efficient in the LF range for constructing reduced models cannot be used in the MF range for general three-dimensional structures. It should be noted that it is essential to have intrisinc reduced models in the MF range (as we have in the LF range) for calculating the MF responses to any multiple loads constituted of deterministic and random excitations. This is the reason why a reduced model method in the MF range has been recently proposed for general dissipative structural-dynamics systems ${ }^{1,2}$. In this method, the reduced model is constructed using the Ritz-Galerkin projection of the variational formulation of the boundary value problem on the dominant eigensubspace of the energy operator of the structure over the medium-frequency band of analysis. Similarly to the LF reduced models, the efficiency of the proposed MF reduced model is due to the small number of generalized dynamical degrees of freedom used in the representation and in addition, is obtained by solving a well-stated generalized symmetric eigenvalue problem for which only the first eigenvalues and the corresponding eigenfunctions of the energy operator related to the MF band have to be calculated. This means that the first normal modes of the LF range are replaced by the first eigenfunctions of the energy operator in the MF range. Finally, it should be noted that the efficiency of such a reduced model approach in the MF range can be very high with respect to any direct approaches validated in the MF range as soon as the MF responses have to be calculated for a large number of multiple deterministic and random loads, particularly for random excitations.

In this paper, we apply and adapt this method for constructing a reduced model in the MF range to a general three-dimensional dissipative structure consisting of an anisotropic, inhomogeneous, viscoelastic bounded medium coupled with an internal acoustic cavity filled with a gas or a liquid. Two fundamental cases are considered: 1) both the structure and the internal acoustic cavity have a medium-frequency "behavior" in the frequency band of analysis; 2) the structure has a medium-frequency "behavior" in the frequency band of analysis while the internal acoustic cavity has a low-frequency "behavior".

In Section I, we present the boundary value problem to be solved in the frequency domain and we recall its variational formulation. In Section II, we introduce a finite dimension approximation. Section III concerns the construction of a vector basis for the reduced model of the structure and the internal acoustic cavity in the MF range. We will see the role played by the low-frequency structural modes on the acoustic cavity response in the MF range. As a 
consequence, we deduce a structural vector basis adapted to the MF range when the structure is coupled with an internal acoustic cavity. Section IV is devoted to the construction of the reduced model while Section V deals with the construction of the dominant eigensubspaces. In Section VI, we present the time-stationary random response using the reduced model. Finally, we present a validation for the two fundamental cases introduced above.

\section{BOUNDARY VALUE PROBLEM AND ITS VARIATIONAL FORMULATION}

\section{A. Definition of the boundary value problem}

We consider linear vibrations (formulated in the frequency domain $\omega$ ) of a three-dimensional structural-acoustic system around a static equilibrium configuration considered as a natural state at rest (see Fig. 1). Let $\Omega_{1}$ be the three-dimensional bounded domain occupied by the structure and made of viscoelastic material. Let $\partial \Omega_{1}=\Gamma_{0} \cup \Gamma_{1} \cup \Gamma_{2}$ be its boundary and $\mathbf{n}_{1}=$ $\left(n_{1,1}, n_{1,2}, n_{1,3}\right)$ be its outward unit normal. Let $\mathbf{u}(\mathbf{x}, \omega)=\left(u_{1}(\mathbf{x}, \omega), u_{2}(\mathbf{x}, \omega), u_{3}(\mathbf{x}, \omega)\right)$ be the displacement field in each point $\mathbf{x}=\left(x_{1}, x_{2}, x_{3}\right)$ in Cartesian coordinates and at frequency $\omega$. On part $\Gamma_{0}$ of the boundary, the structure is fixed $(\mathbf{u}=\mathbf{0})$ whereas on part $\Gamma_{1} \cup \Gamma_{2}$ it is free. The structure is coupled with an internal dissipative acoustic fluid (gas or liquid) occupying the three-dimensional bounded domain $\Omega_{2}$ whose boundary $\partial \Omega_{2}$ is the coupling interface $\Gamma_{2}$. The outward unit normal to $\partial \Omega_{2}$ is denoted as $\mathbf{n}_{2}=\left(n_{2,1}, n_{2,2}, n_{2,3}\right)$ and we have $\mathbf{n}_{2}=-\mathbf{n}_{1}$. We denote the pressure field in $\Omega_{2}$ as $p(\mathbf{x}, \omega)$. We introduce a narrow MF band $B$ such that

$$
B=\left[\omega_{B}-\Delta \omega / 2, \omega_{B}+\Delta \omega / 2\right],
$$

in which $\omega_{B}$ is the center frequency and $\Delta \omega$ is the bandwidth such that $\Delta \omega / \omega_{B} \ll 1$ and $\omega_{B}>\Delta \omega / 2$. With $B$ we associate interval

$$
\widetilde{B}=\left[-\omega_{B}-\Delta \omega / 2,-\omega_{B}+\Delta \omega / 2\right]
$$

The structure is submitted to a square integrable surface force field $\mathbf{x} \mapsto \eta(\omega) \mathbf{g}(\mathbf{x}, \omega)$ from $\Gamma_{1}$ into $\mathbb{C}^{3}$, in which $\eta(\omega)$ is a function from $\mathbb{R}$ into $\mathbb{C}$, such that $\eta(\omega)=0$ if $\omega$ is not in $B \cup \widetilde{B}$, continuous on $B$, verifying $|\eta(-\omega)|=|\eta(\omega)|$ and such that $|\eta(\omega)| \neq 0$ for all $\omega$ in $B$.

For this structural-acoustic system, we use the model and the boundary value problem developed for the MF range in Chapter XIV of Ref. 3. Introducing components $\left(g_{1}, g_{2}, g_{3}\right)$ of $\mathbf{g}$, the boundary value problem for the structure is written as follows in terms of $\mathbf{u}$ (the convention for the Fourier transform being $v(\omega)=\int_{\mathbb{R}} e^{-i \omega t} v(t) d t$,

$$
\begin{gathered}
-\omega^{2} \rho_{1} u_{i}-\sigma_{i j, j}=0 \quad \text { in } \Omega_{1}, \\
\sigma_{i j} n_{1, j}=\eta g_{i} \quad \text { on } \quad \Gamma_{1}, \\
\sigma_{i j} n_{1, j}=-p n_{1, j} \quad \text { on } \quad \Gamma_{2}, \\
u_{i}=0 \quad \text { on } \quad \Gamma_{0},
\end{gathered}
$$

in which $i=1,2,3$, where the summation over index $j$ is used, and where $\rho_{1}(\mathbf{x})>0$ is the mass density of the structure and $\sigma_{i j, j}=\sum_{j=1}^{3} \partial \sigma_{i j} / \partial x_{j}$. For a linear viscoelastic material, stress tensor $\sigma_{i j}$ is written as

$$
\sigma_{i j}=\mathbb{a}_{i j k h}(\mathbf{x}, \omega) \varepsilon_{k h}(\mathbf{u})+\mathfrak{b}_{i j k h}(\mathbf{x}, \omega) \varepsilon_{k h}(i \omega \mathbf{u})
$$

J. Acoust. Soc. Am., 106(6), 3362-3374 (1999). 
in which the summation over indices $k$ and $h$ is used and where $\varepsilon_{k h}(\mathbf{u})=\left(\partial u_{k} / \partial x_{h}+\right.$ $\left.\partial u_{h} / \partial x_{k}\right) / 2$ is the linearized strain tensor. Coefficients $\mathbb{a}_{i j k h}(\mathbf{x}, \omega)$ and $\mathrm{b}_{i j k h}(\mathbf{x}, \omega)$ are real, depend on $\mathbf{x}$ and $\omega$, verify the usual properties of symmetry and positiveness ${ }^{3-6}$ and are such that $\mathbb{a}_{i j k h}(\mathbf{x},-\omega)=\mathbb{a}_{i j k h}(\mathbf{x}, \omega)$ and $\mathfrak{b}_{i j k h}(\mathbf{x},-\omega)=\mathfrak{b}_{i j k h}(\mathbf{x}, \omega)$.

Concerning the internal dissipative acoustic fluid, the pressure in the fluid is written (see Chapter XIV of Ref. 3) as

$$
p(\mathbf{x}, \omega)=-i \omega \rho_{2} \psi(\mathbf{x}, \omega)-\kappa \pi_{2}(\mathbf{u}) \quad \text { in } \quad \Omega_{2} \cup \Gamma_{2},
$$

in which $\kappa$ is a positive constant such that

$$
\kappa=\frac{\rho_{2} c_{2}^{2}}{\left|\Omega_{2}\right|} \quad, \quad\left|\Omega_{2}\right|=\int_{\Omega_{2}} d \mathbf{x},
$$

where $\rho_{2}>0$ and $c_{2}$ are the constant mass density and the constant speed of sound of the acoustic fluid at equilibrium and where $\pi_{2}(\mathbf{u})$ is defined by

$$
\pi_{2}(\mathbf{u})=\int_{\Gamma_{2}} \mathbf{u}(\mathbf{x}, \omega) \cdot \mathbf{n}_{2}(\mathbf{x}) d s(\mathbf{x})
$$

The new unknown field $\psi(\mathbf{x}, \omega)$ is related to the velocity field $\mathbf{v}(\mathbf{x}, \omega)$ of the dissipative acoustic fluid by the equation $\mathbf{v}(\mathbf{x}, \omega)=(1+i \omega \tau) \boldsymbol{\nabla} \psi(\mathbf{x}, \omega)$ in which $\tau$ is a constant coefficient ${ }^{3}$ related to the viscosity of the acoustic fluid ( $\tau$ may depend on frequency $\omega$ ). The boundary value problem for the internal acoustic fluid is written as follows in terms of field $\psi$

$$
\begin{gathered}
-\omega^{2} \frac{\rho_{2}}{c_{2}^{2}} \psi(\mathbf{x}, \omega)-i \omega \tau \rho_{2} \nabla^{2} \psi(\mathbf{x}, \omega)-\rho_{2} \nabla^{2} \psi(\mathbf{x}, \omega)=-\frac{i \omega \kappa}{c_{2}^{2}} \pi_{2}(\mathbf{u}) \quad \text { in } \Omega_{2} \\
\rho_{2}(1+i \omega \tau) \frac{\partial \psi}{\partial \mathbf{n}_{2}}=i \omega \rho_{2} \mathbf{u} \cdot \mathbf{n}_{2} \quad \text { on } \quad \Gamma_{2} \\
\int_{\Omega_{2}} \psi(\mathbf{x}, \omega) d \mathbf{x}=0
\end{gathered}
$$

The boundary value problem of the structural-acoustic problem is defined by Eqs. (3) to (13).

\section{B. Variational formulation}

Let $V_{1}$ be the space of admissible displacement fields $\mathbf{u}$ defined on $\Omega_{1}$ with values in $\mathbb{C}^{3}$ such that $\mathbf{u}=\mathbf{0}$ on $\Gamma_{0}$. Let $V_{2}$ be the space of admissible fields $\psi$ defined on $\Omega_{2}$ with values in $\mathbb{C}$ such that $\int_{\Omega_{2}} \psi(\mathbf{x}) d \mathbf{x}=0$. Below, $\bar{z}$ denotes the conjugate of the complex number $z$. The variational formulation of the boundary value problem in $\mathbf{u}$ and $\psi$ defined by Eqs. (3) to (13) is obtained using the test-function method and is expressed as follows (see Chapters II and XIV of Ref. 3). For all $\omega$ in $B \cup \widetilde{B}$, find $\{\mathbf{u}(\omega), \psi(\omega)\}$ in $V_{1} \times V_{2}$ such that, for all $\{\mathbf{v}, \phi\}$ in $V_{1} \times V_{2}$,

$$
\begin{gathered}
a_{1}(\mathbf{u}, \mathbf{v} ; \omega)+\kappa j(\mathbf{u}, \mathbf{v})+i \omega c(\psi, \mathbf{v})=f(\mathbf{v} ; \omega), \\
i \omega \overline{c(\phi, \mathbf{u})}-a_{2}(\psi, \phi ; \omega)=0,
\end{gathered}
$$

in which $f(\mathbf{v} ; \omega)$ is defined by

$$
f(\mathbf{v} ; \omega)=\eta(\omega) \int_{\Gamma_{1}} \mathbf{g}(\mathbf{x}, \omega) \cdot \overline{\mathbf{v}(\mathbf{x})} d s(\mathbf{x}),
$$

J. Acoust. Soc. Am., 106(6), 3362-3374 (1999). 
and where $a_{1}(\mathbf{u}, \mathbf{v} ; \omega)$ is written as

$$
\begin{gathered}
a_{1}(\mathbf{u}, \mathbf{v} ; \omega)=-\omega^{2} m_{1}(\mathbf{u}, \mathbf{v})+i \omega d_{1}(\mathbf{u}, \mathbf{v} ; \omega)+k_{1}(\mathbf{u}, \mathbf{v} ; \omega) \\
m_{1}(\mathbf{u}, \mathbf{v})=\int_{\Omega_{1}} \rho_{1}(\mathbf{x}) \mathbf{u}(\mathbf{x}, \omega) \cdot \overline{\mathbf{v}(\mathbf{x})} d \mathbf{x} \\
d_{1}(\mathbf{u}, \mathbf{v} ; \omega)=\int_{\Omega_{1}} \mathbb{b}_{i j k h}(\mathbf{x}, \omega) \varepsilon_{k h}(\mathbf{u}) \varepsilon_{i j}(\overline{\mathbf{v}}) d \mathbf{x} \\
k_{1}(\mathbf{u}, \mathbf{v} ; \omega)=\int_{\Omega_{1}} \mathbb{a}_{i j k h}(\mathbf{x}, \omega) \varepsilon_{k h}(\mathbf{u}) \varepsilon_{i j}(\overline{\mathbf{v}}) d \mathbf{x}
\end{gathered}
$$

in which the summation over indices $i, j, k$ and $h$ is used. It is assumed that $d_{1}(\mathbf{u}, \mathbf{v} ; \omega)$ and $k_{1}(\mathbf{u}, \mathbf{v} ; \omega)$ are continuous functions on band $B$ with respect to $\omega$. In Eqs. (14) and (15), we have

$$
\begin{gathered}
c(\psi, \mathbf{v})=\rho_{2} \int_{\Gamma_{2}} \psi(\mathbf{x}, \omega) \mathbf{n}_{2}(\mathbf{x}) \cdot \overline{\mathbf{v}(\mathbf{x})} d s(\mathbf{x}) \\
j(\mathbf{u}, \mathbf{v})=\pi_{2}(\mathbf{u}) \pi_{2}(\overline{\mathbf{v}}) \\
a_{2}(\psi, \phi ; \omega)=-\omega^{2} m_{2}(\psi, \phi)+i \omega d_{2}(\psi, \phi ; \omega)+k_{2}(\psi, \phi) \\
m_{2}(\psi, \phi)=\frac{\rho_{2}}{c_{2}^{2}} \int_{\Omega_{2}} \psi(\mathbf{x}, \omega) \overline{\phi(\mathbf{x})} d \mathbf{x} \\
d_{2}(\psi, \phi ; \omega)=\tau(\omega) k_{2}(\psi, \phi) \\
k_{2}(\psi, \phi)=\rho_{2} \int_{\Omega_{2}} \nabla \psi(\mathbf{x}, \omega) \cdot \nabla \overline{\phi(\mathbf{x})} d \mathbf{x}
\end{gathered}
$$

For all $\omega$ in $B \cup \widetilde{B}$, the problem defined by Eqs. (14) and (15) has a unique solution $\{\mathbf{u}(\omega), \psi(\omega)\}$ in $V_{1} \times V_{2}$.

\section{FINITE DIMENSION APPROXIMATION}

The finite dimension approximation of the problem defined by Eqs. (14) and (15) is obtained by using the Ritz-Galerkin method. We then consider a complete family of independent $\mathbb{R}^{3}$-valued functions $\left\{\mathbf{u}_{\alpha}\right\}_{\alpha \geq 1}$ in admissible space $V_{1}$ and a complete family of independent real-valued functions $\left\{\psi_{\beta}\right\}_{\beta \geq 1}$ in admissible space $V_{2}$. We consider 1) a subspace $V_{1, n_{1}} \subset V_{1}$ of finite dimension $n_{1} \geq 1$ spanned by the family $\left\{\mathbf{u}_{1}, \ldots, \mathbf{u}_{n_{1}}\right\}$ and 2) a subspace $V_{2, n_{2}} \subset V_{2}$ of finite dimension $n_{2} \geq 1$ spanned by the family $\left\{\psi_{1}, \ldots, \psi_{n_{2}}\right\}$. In practice, each family can be either a finite element basis ${ }^{7,8}$ associated with a finite element mesh of domain $\Omega_{1}$ for $\left\{\mathbf{u}_{\alpha}\right\}_{\alpha}$ and domain $\Omega_{2}$ for $\left\{\psi_{\beta}\right\}_{\beta}$, or, for particular cases corresponding to simple shapes of geometry, any functional basis constructed in the context of an analytical approach such as a sequence of structural normal modes ${ }^{9,10}$ of the structure in vacuo and a sequence of acoustic modes $^{9,11}$ of the internal acoustic cavity with a rigid wall. Let $\{\mathbf{u}(\omega), \psi(\omega)\} \in V_{1} \times V_{2}$ be the solution of Eqs. (14) and (15). Its projection on $V_{1, n_{1}} \times V_{2, n_{2}}$ is written as

$$
\widetilde{\mathbf{u}}(\mathbf{x}, \omega)=\sum_{\alpha=1}^{n_{1}} q_{1, \alpha}(\omega) \mathbf{u}_{\alpha}(\mathbf{x})
$$

J. Acoust. Soc. Am., 106(6), 3362-3374 (1999). 


$$
\widetilde{\psi}(\mathbf{x}, \omega)=\sum_{\beta=1}^{n_{2}} q_{2, \beta}(\omega) \psi_{\beta}(\mathbf{x})
$$

From Eqs. (14) and (15), we deduce that $\mathbf{q}_{1}(\omega)=\left(q_{1,1}(\omega), \ldots, q_{1, n_{1}}(\omega)\right) \in \mathbb{C}^{n_{1}}$ and $\mathbf{q}_{2}(\omega)=\left(q_{2,1}(\omega), \ldots, q_{2, n_{2}}(\omega)\right) \in \mathbb{C}^{n_{2}}$ is the unique solution of the linear equation ${ }^{3}$

$$
\left[\begin{array}{cc}
{\left[A_{1}(\omega)\right]+\kappa[J]} & i \omega[C] \\
i \omega[C]^{T} & -\left[A_{2}(\omega)\right]
\end{array}\right]\left[\begin{array}{l}
\mathbf{q}_{1}(\omega) \\
\mathbf{q}_{2}(\omega)
\end{array}\right]=\left[\begin{array}{c}
\eta(\omega) \mathbf{F}(\omega) \\
0
\end{array}\right]
$$

In Eq. (29), $\left[A_{1}(\omega)\right]$ is an $\left(n_{1} \times n_{1}\right)$ symmetric complex matrix (invertible for all $\omega$ in $B \cup \widetilde{B}$ ) which is written as

$$
\left[A_{1}(\omega)\right]=-\omega^{2}\left[M_{1}\right]+i \omega\left[D_{1}(\omega)\right]+\left[K_{1}(\omega)\right]
$$

in which $\left[M_{1}\right],\left[D_{1}(\omega)\right]$ and $\left[K_{1}(\omega)\right]$ are positive-definite symmetric $\left(n_{1} \times n_{1}\right)$ real matrices such that $\left[M_{1}\right]_{\alpha \alpha^{\prime}}=m_{1}\left(\mathbf{u}_{\alpha^{\prime}}, \mathbf{u}_{\alpha}\right),\left[D_{1}(\omega)\right]_{\alpha \alpha^{\prime}}=d_{1}\left(\mathbf{u}_{\alpha^{\prime}}, \mathbf{u}_{\alpha} ; \omega\right)$ and $\left[K_{1}(\omega)\right]_{\alpha \alpha^{\prime}}=$ $k_{1}\left(\mathbf{u}_{\alpha^{\prime}}, \mathbf{u}_{\alpha} ; \omega\right)$. Vector $\mathbf{F}(\omega)=\left(F_{1}(\omega), \ldots, F_{n_{1}}(\omega)\right) \in \mathbb{C}^{n_{1}}$ is such that

$$
\eta(\omega) F_{\alpha}(\omega)=f\left(\mathbf{u}_{\alpha} ; \omega\right)
$$

Matrix $[J]$ is an $\left(n_{1} \times n_{1}\right)$ symmetric real matrix which can be written as

$$
[J]=\Pi_{2} \Pi_{2}^{T},
$$

in which $\Pi_{2}=\left(\Pi_{2,1}, \ldots, \Pi_{2, n_{1}}\right) \in \mathbb{R}^{n_{1}}$ is such that

$$
\Pi_{2, \alpha}=\pi_{2}\left(\mathbf{u}_{\alpha}\right)
$$

In Eq. (29), $\left[A_{2}(\omega)\right]$ is an $\left(n_{2} \times n_{2}\right)$ symmetric complex matrix (invertible for all real $\omega$ in $B \cup \widetilde{B})$ which is written as

$$
\left[A_{2}(\omega)\right]=-\omega^{2}\left[M_{2}\right]+i \omega\left[D_{2}(\omega)\right]+\left[K_{2}\right]
$$

in which $\left[M_{2}\right],\left[D_{2}(\omega)\right]$ and $\left[K_{2}\right]$ are positive-definite symmetric $\left(n_{2} \times n_{2}\right)$ real matrices such that $\left[M_{2}\right]_{\beta \beta^{\prime}}=m_{2}\left(\psi_{\beta^{\prime}}, \psi_{\beta}\right),\left[D_{2}(\omega)\right]=\tau(\omega)\left[K_{2}\right]$ and $\left[K_{2}\right]_{\beta \beta^{\prime}}=k_{2}\left(\psi_{\beta^{\prime}}, \psi_{\beta}\right)$. It should be noted that $\left[K_{2}\right]$ is positive definite due to the constraint $\int_{\Omega_{2}} \psi d \mathbf{x}=0$ which is included in space $V_{2}$. In the context of use of the finite element method, if this constraint is not included in the construction of $\left\{\psi_{\beta}\right\}_{\beta}$, then $\left[K_{2}\right]$ is only positive and the constraint $\mathbf{L}^{T} \mathbf{q}_{2}=0$ corresponding to the finite element discretization of $\int_{\Omega_{2}} \psi d \mathbf{x}=0$ has to be added to Eq. (29). Finally, in Eq. (29), $[C]$ is an $\left(n_{1} \times n_{2}\right)$ real matrix such that

$$
[C]_{\alpha \beta}=c\left(\psi_{\beta}, \mathbf{u}_{\alpha}\right)
$$

The approximation of pressure field $p$ defined by Eq. (8) is written as $\widetilde{p}(\mathbf{x}, \omega)=-i \omega \rho_{2} \widetilde{\psi}(\mathbf{x}, \omega)$ $-\kappa \pi_{2}(\widetilde{\mathbf{u}})$, i.e.,

$$
\widetilde{p}(\mathbf{x}, \omega)=-i \omega \rho_{2} \boldsymbol{\Psi}(\mathbf{x})^{T} \mathbf{q}_{2}(\omega)-\kappa \boldsymbol{\Pi}_{2}^{T} \mathbf{q}_{1}(\omega),
$$

in which $\boldsymbol{\Psi}(\mathbf{x})=\left(\psi_{1}(\mathbf{x}), \ldots, \psi_{n_{2}}(\mathbf{x})\right) \in \mathbb{R}^{n_{2}}$. 


\section{CONSTRUCTION OF A VECTOR BASIS FOR THE REDUCED MODEL}

\section{A. Vector bases adapted to MF band $B$ for the structure in vacuo and the internal acoustic cavity with rigid wall}

Two vector bases adapted to MF band $B$ can be constructed for the structure in vacuo and the internal acoustic cavity with rigid wall by applying the method presented in Refs. 1 and 2 (for the details, we refer the reader to these references). These two vector bases correspond to the dominant eigensubspaces of the energy operators relative to band $B$ for the structure in vacuo and the internal acoustic fluid with rigid wall. In the context of the finite dimension approximation introduced in Section II, the procedure can be summarized as follows. Let $a=1$ or $a=2$ be the index related to the structure or the internal acoustic fluid. Let $N_{a} \ll n_{a}$ be the order of the reduced model related to the structure $(a=1)$ or the internal acoustic fluid $(a=2)$. Let $\left[P_{a}\right]$ be the $\left(n_{a} \times N_{a}\right)$ real matrix whose columns are the $N_{a}$ eigenvectors $\left\{\mathbf{P}_{a}^{1}, \ldots, \mathbf{P}_{a}^{N_{a}}\right\}$ corresponding to the $N_{a}$ highest eigenvalues $\lambda_{a}^{1} \geq \ldots \geq \lambda_{a}^{N_{a}}$ of the generalized symmetric eigenvalue problem

$$
\left[H_{a}\right]\left[P_{a}\right]=\left[G_{a}\right]\left[P_{a}\right]\left[\Lambda_{a}\right],
$$

such that

$$
\begin{aligned}
& {\left[P_{a}\right]^{T}\left[G_{a}\right]\left[P_{a}\right]=\left[I_{a}\right],} \\
& {\left[P_{a}\right]^{T}\left[H_{a}\right]\left[P_{a}\right]=\left[\Lambda_{a}\right],}
\end{aligned}
$$

in which $\left[I_{a}\right]$ is the $\left(N_{a} \times N_{a}\right)$ identity matrix, $\left[\Lambda_{a}\right]$ is the $\left(N_{a} \times N_{a}\right)$ diagonal matrix of eigenvalues $\lambda_{a}^{1}, \ldots, \lambda_{a}^{N_{a}}$ and where $\left[G_{a}\right]$ and $\left[H_{a}\right]$ are positive-definite symmetric $\left(n_{a} \times n_{a}\right)$ real matrices such that

$$
\begin{gathered}
{\left[G_{1}\right]_{\alpha \alpha^{\prime}}=\int_{\Omega_{1}} \mathbf{u}_{\alpha^{\prime}} \cdot \mathbf{u}_{\alpha} d \mathbf{x} \quad, \quad\left[G_{2}\right]_{\beta \beta^{\prime}}=\int_{\Omega_{2}} \psi_{\beta^{\prime}} \cdot \psi_{\beta} d \mathbf{x},} \\
{\left[H_{a}\right]=\left[G_{a}\right]\left[E_{a}\right]\left[G_{a}\right] .}
\end{gathered}
$$

In Eq. (40), positive-definite symmetric $\left(n_{a} \times n_{a}\right)$ real matrix $\left[E_{a}\right]$ is the projection of the energy operator of the structure $(a=1)$ or the internal acoustic fluid $(a=2)$, such that

$$
\begin{gathered}
{\left[E_{a}\right]=\int_{B}\left[e_{a}(\omega)\right] d \omega,} \\
{\left[e_{a}(\omega)\right]=\frac{1}{\pi} \omega^{2}|\eta(\omega)|^{2} \Re e\left\{\left[T_{a}(\omega)\right]^{*}\left[M_{a}\right]\left[T_{a}(\omega)\right]\right\},} \\
{\left[T_{a}(\omega)\right]=\left[A_{a}(\omega)\right]^{-1} \quad ; \quad\left[T_{a}(\omega)\right]^{*}=\overline{\left[T_{a}(\omega)\right]^{T}}=\overline{\left[T_{a}(\omega)\right]},}
\end{gathered}
$$

in which $\left[A_{a}(\omega)\right]$ is defined by Eq. (30) for $a=1$ and Eq. (33) for $a=2$.

\section{B. Remark concerning the acoustic vector basis relative to MF band $B$}

It can easily be proved that for the internal acoustic fluid $(a=2)$, eigenvectors $\left\{\mathbf{P}_{2}^{1}, \ldots, \mathbf{P}_{2}^{N_{2}}\right\}$, associated with the $N_{2}$ highest eigenvalues of generalized symmetric eigenvalue problem $\left[H_{2}\right]\left[P_{2}\right]=\left[G_{2}\right]\left[P_{2}\right]\left[\Lambda_{2}\right]$ relative to MF band $B$, coincide with the finite dimension approximation of the acoustic modes of the internal acoustic cavity with rigid wall, whose 
eigenfrequencies are in MF band $B$. This particular property is due to the fact that two conditions are simultaneously satisfied: mass density $\rho_{2}$ of the acoustic fluid is a constant and damping sesquilinear form $d_{2}(\psi, \phi ; \omega)=\tau(\omega) k_{2}(\psi, \phi)$ is diagonalized by the acoustic modes. If either of these two conditions is not satisfied, then this particular property does not hold; generally, these conditions are not satisfied for structures in the MF range. It should be noted that, in the context of a finite element model of the internal acoustic fluid, if the rank of the acoustic modes belonging to MF band $B$ is high (case of MF behavior of the internal acoustic fluid), then the proposed method is an efficient tool for computation of these acoustic modes. Conversely, if the rank of the acoustic modes belonging to MF band $B$ is low (case of LF behavior of the internal acoustic fluid), then the usual numerical methods for computation of the acoustic modes (such as subspace iteration or Lanczos methods ${ }^{12-15}$ ) are more efficient.

\section{Role played by the low-frequency structural modes on the acoustic cavity response in MF band $B$}

In this section, in order to analyze this role, we introduce particular assumptions for simplifying the explanations. Let us assume that $\left\{\psi_{\beta}\right\}_{\beta}$ corresponds to the acoustic modes verifying

$$
k_{2}\left(\psi_{\beta}, \phi\right)=\omega_{2, \beta}^{2} m_{2}\left(\psi_{\beta}, \phi\right) \quad, \quad \forall \phi \in V_{2} \quad,
$$

with the normalization $m_{2}\left(\psi_{\beta}, \psi_{\beta}\right)=1$ and such that $\left[D_{2}(\omega)\right]_{\beta \beta^{\prime}}=2 \xi_{2, \beta} \omega_{2, \beta} \delta_{\beta \beta^{\prime}}$ in which $\xi_{2, \beta}>0$ is an acoustic damping rate depending on $\beta$ but independent of $\omega$. We choose $\left\{\mathbf{u}_{\alpha}\right\}_{\alpha}=\left\{\widehat{\mathbf{u}}_{\alpha}\right\}_{\alpha}$ as the structural modes taking into account the additional stiffness term $\kappa j(\mathbf{u}, \mathbf{v})$. Consequently, the spectral problem is written as

$$
\widehat{k}_{1}\left(\widehat{\mathbf{u}}_{\alpha}, \mathbf{v}\right)=\widehat{\omega}_{1, \alpha}^{2} m_{1}\left(\widehat{\mathbf{u}}_{\alpha}, \mathbf{v}\right) \quad, \quad \forall \mathbf{v} \in V_{1} \quad,
$$

in which $\widehat{k}_{1}(\mathbf{u}, \mathbf{v})=k_{1}(\mathbf{u}, \mathbf{v} ; 0)+\kappa j(\mathbf{u}, \mathbf{v})$. The normalization is such that $m_{1}\left(\widehat{\mathbf{u}}_{\alpha}, \widehat{\mathbf{u}}_{\alpha}\right)=1$ and it is assumed that $\left[D_{1}(\omega)\right]_{\alpha \alpha^{\prime}}=2 \widehat{\xi}_{1, \alpha} \widehat{\omega}_{1, \alpha} \delta_{\alpha \alpha^{\prime}}$ where $\widehat{\xi}_{1, \alpha}>0$ is a structural damping rate depending on $\alpha$ but independent of $\omega$. From Eqs. (29) to (34), we deduce that $\mathbf{q}_{2}(\omega)$ is the solution of the linear matrix equation

$$
\left[\mathbb{A}_{2}(\omega)\right] \mathbf{q}_{2}(\omega)=\mathbb{F}_{2}(\omega),
$$

in which $\mathbb{F}_{2}(\omega)$ is a given vector in $\mathbb{C}^{n_{2}}$ and $\left[\mathbb{A}_{2}(\omega)\right]$ is an $\left(n_{2} \times n_{2}\right)$ symmetric complex matrix which can be written as $\left[\mathbb{A}_{2}(\omega)\right]=\left[\mathbb{A}_{2}^{F}(\omega)\right]+\left[\mathbb{A}_{2}^{S}(\omega)\right]$ where, for all $\beta$ and $\beta^{\prime}$ in $\left\{1, \ldots, n_{2}\right\}$,

$$
\begin{aligned}
& {\left[\AA_{2}^{F}(\omega)\right]_{\beta \beta^{\prime}}=\left(-\omega^{2}+2 i \omega \omega_{2, \beta} \xi_{2, \beta}+\omega_{2, \beta}^{2}\right) \delta_{\beta \beta^{\prime}},} \\
& {\left[\mathbb{A}_{2}^{S}(\omega)\right]_{\beta \beta^{\prime}}=-\sum_{\alpha=1}^{n_{1}} \frac{\omega^{2} C_{\alpha \beta} C_{\alpha \beta^{\prime}}}{-\omega^{2}+2 i \omega \widehat{\omega}_{1, \alpha} \widehat{\xi}_{1, \alpha}+\widehat{\omega}_{1, \alpha}^{2}} .}
\end{aligned}
$$

Let us consider the MF response of the acoustic cavity due to an acoustic mode $\psi_{\beta}$ whose eigenfrequency is $\omega_{2, \beta}$. Consequently, $\omega$ and $\omega_{2, \beta}$ belong to MF band $B$. Let us investigate the contribution of the first $M$ low-frequency structural modes (whose eigenfrequencies $\widehat{\omega}_{1,1}, \ldots, \widehat{\omega}_{1, M}$ belong to the low-frequency range) to the acoustic mode $\psi_{\beta}$ whose eigenfrequency is $\omega_{2, \beta}$ belonging to MF band $B$. We then have

$$
0<\widehat{\omega}_{1,1} \leq \ldots \leq \widehat{\omega}_{1, M} \ll \omega \in B \quad,
$$

J. Acoust. Soc. Am., 106(6), 3362-3374 (1999). 
and therefore, the contribution in $\left[A_{2}(\omega)\right]_{\beta \beta}$ of structural mode $\widehat{\mathbf{u}}_{\alpha}$ whose eigenfrequency $\widehat{\omega}_{1, \alpha}$ is such that $\widehat{\omega}_{1, \alpha} \ll \omega \in B$, is equal to

$$
\frac{-\omega^{2} C_{\alpha \beta}^{2}}{-\omega^{2}+2 i \omega \widehat{\omega}_{1, \alpha} \widehat{\xi}_{1, \alpha}+\widehat{\omega}_{1, \alpha}^{2}} \simeq C_{\alpha \beta}^{2}
$$

This positive term $C_{\alpha \beta}^{2}$ contributes to increase the value of the acoustic eigenfrequency $\omega_{2, \beta}$ because generalized stiffness term $\omega_{2, b}^{2}$ in $\left[\mathbb{A}_{2}(\omega)\right]_{\beta \beta}$ is increasing of positive term $C_{\alpha \beta}^{2}$ for each structural mode $\widehat{\mathbf{u}}_{\alpha}$ such that $\widehat{\omega}_{1, \alpha} \ll \omega \in B$. Consequently, the rate of convergence of the internal acoustic MF response can be increased by "adding" the lowest structural modes $\left\{\widehat{\mathbf{u}}_{1}, \ldots, \widehat{\mathbf{u}}_{M}\right\}$ (or equivalently the lowest structural modes $\left\{\mathbf{u}_{1}, \ldots, \mathbf{u}_{M}\right\}$ of the structure in vacuo) to vectors $\left\{\mathbf{P}_{1}^{1}, \ldots, \mathbf{P}_{1}^{N_{1}}\right\}$ adapted to the prediction of the structural response in MF band $B$.

\section{Structural vector basis adapted to MF band $B$ for prediction of the internal acoustic response in MF band $B$}

Taking into account the conclusion of Section III.C, MF band $B$ being fixed, we consider eigenvectors $\left\{\mathbf{P}_{1}^{1}, \ldots, \mathbf{P}_{1}^{N_{1}}\right\}$ introduced in Section III.A, such that (see Eq. (37)),

$$
\mathbf{P}_{1}^{k^{T}}\left[G_{1}\right] \mathbf{P}_{1}^{j}=\delta_{j k} \quad, \quad j \quad \text { and } \quad k \quad \text { in }\left\{1, \ldots, N_{1}\right\}
$$

and we consider the $N_{0}$ structural modes $\left\{\mathbf{Q}_{1}^{1}, \ldots, \mathbf{Q}_{1}^{N_{0}}\right\}$ corresponding to the lowest structural eigenfrequencies $0<\omega_{1,1} \leq \ldots \leq \omega_{1, N_{0}}$ such that, for all $\alpha$ in $\left\{1, \ldots, N_{0}\right\}$,

$$
\left[K_{1}(0)\right] \mathbf{Q}_{1}^{\alpha}=\omega_{1, \alpha}^{2}\left[M_{1}\right] \mathbf{Q}_{1}^{\alpha}
$$

We assume that

$$
N_{1}^{S}=N_{1}+N_{0}<n_{1},
$$

and that vectors $\left\{\mathbf{P}_{1}^{1}, \ldots, \mathbf{P}_{1}^{N_{1}}, \mathbf{Q}_{1}^{1}, \ldots, \mathbf{Q}_{1}^{N_{0}}\right\}$ constitute a set of linearly independent vectors in $\mathbb{R}^{n_{1}}$. In practice, $M$ structural modes $\left\{\mathbf{Q}_{1}^{\alpha_{1}}, \ldots, \mathbf{Q}_{1}^{\alpha_{M}}\right\}$ are considered, the $\left(n_{1} \times\left(N_{1}+\right.\right.$ $M)$ ) real matrix $[X]=\left[\mathbf{P}_{1}^{1} \ldots \mathbf{P}_{1}^{N_{1}} \mathbf{Q}_{1}^{\alpha_{1}} \ldots \mathbf{Q}_{1}^{\alpha_{M}}\right]$ is constructed and finally, the $[Q][R]$ factorization of matrix $[X]$ is computed in which $[Q]$ is an $\left(n_{1} \times n_{1}\right)$ orthogonal matrix and $[R]$ is an $\left(n_{1} \times\left(N_{1}+M\right)\right)$ real upper triangular matrix. If none of the diagonal elements of $[R]$ is equal to zero, then $N_{0}=M$; if there are $m_{0}$ diagonal elements equal to zero, then $N_{0}=M-m_{0}$ and the corresponding structural modes $\mathbf{Q}_{1}^{\alpha_{j}}$ are eliminated. We then deduce the set $\left\{\mathbf{Q}_{1}^{1}, \ldots, \mathbf{Q}_{1}^{N_{0}}\right\}$. Finally, a Gram-Schmidt algorithm with respect to the inner product defined by matrix $\left[G_{1}\right]$ is applied to the set $\left\{\mathbf{P}_{1}^{1}, \ldots, \mathbf{P}_{1}^{N_{1}}, \mathbf{Q}_{1}^{1}, \ldots, \mathbf{Q}_{1}^{N_{0}}\right\}$ for constructing the $N_{1}^{S}$ linearly independent vectors $\left\{\mathbf{P}_{1}^{1}, \ldots, \mathbf{P}_{1}^{N_{1}}, \mathbf{P}_{1}^{N_{1}+1}, \ldots, \mathbf{P}_{1}^{N_{1}+N_{0}}\right\}$ in $\mathbb{R}^{n_{1}}$, such that for $j$ and $k$ in $\left\{1, \ldots, N_{1}^{S}\right\}$, we have $\mathbf{P}_{1}^{k^{T}}\left[G_{1}\right] \mathbf{P}_{1}^{j}=\delta_{j k}$. Vectors $\left\{\mathbf{P}_{1}^{N_{1}+k}, k=1, \ldots, N_{0}\right\}$ are constructed by the recurrence $\mathbf{P}_{1}^{N_{1}+k}=a_{k} \mathbf{W}_{k}$ in which

$$
\begin{gathered}
\mathbf{W}_{k}=\mathbf{Q}_{1}^{k}-\sum_{j=1}^{N_{1}+k-1}\left(\mathbf{P}_{1}^{j^{T}}\left[G_{1}\right] \mathbf{Q}_{1}^{k}\right) \mathbf{P}_{1}^{j}, \\
a_{k}=\left(\mathbf{W}_{k}^{T}\left[G_{1}\right] \mathbf{W}_{k}\right)^{-1} .
\end{gathered}
$$

J. Acoust. Soc. Am., 106(6), 3362-3374 (1999). 
Finally, we introduce the $\left(n_{1} \times N_{1}^{S}\right)$ real matrix $\left[P_{1}^{S}\right]$ such that

$$
\left[P_{1}^{S}\right]=\left[\mathbf{P}_{1}^{1} \ldots \mathbf{P}_{1}^{N_{1}} \mathbf{Q}_{1}^{1} \ldots \mathbf{Q}_{1}^{N_{0}}\right]
$$

It should be noted that if $N_{0}=0$, then $N_{1}^{S}=N_{1}$ and $\left[P_{1}^{S}\right]=\left[P_{1}\right]$.

\section{CONSTRUCTION OF THE REDUCED MODEL ADAPTED TO MF BAND $B$}

The reduced model adapted to MF band $B$ is obtained ${ }^{1,2}$ by introducing the new variable $\boldsymbol{\theta}_{1}(\omega)=\left(\theta_{1,1}(\omega), \ldots, \theta_{1, N_{1}^{S}}(\omega)\right)$ for the structure and the new variable $\boldsymbol{\theta}_{2}(\omega)=$ $\left(\theta_{2,1}(\omega), \ldots, \theta_{2, N_{2}}(\omega)\right)$ for the internal acoustic fluid, such that

$$
\mathbf{q}_{1}(\omega)=\left[P_{1}^{S}\right] \boldsymbol{\theta}_{1}(\omega) \quad ; \quad \mathbf{q}_{2}(\omega)=\left[P_{2}\right] \boldsymbol{\theta}_{2}(\omega) .
$$

From Eqs. (29) and (44), we deduce that for all $\omega$ in $B \cup \widetilde{B},\left\{\boldsymbol{\theta}_{1}(\omega), \boldsymbol{\theta}_{2}(\omega)\right\} \in \mathbb{C}^{N_{1}^{S}} \times \mathbb{C}^{N_{2}}$ is the unique solution of the linear equation

$$
\left[\begin{array}{cc}
{\left[\mathcal{A}_{1}(\omega)\right]+\kappa[\mathcal{J}]} & i \omega[\mathcal{C}] \\
i \omega[\mathcal{C}]^{T} & -\left[\mathcal{A}_{2}(\omega)\right]
\end{array}\right]\left[\begin{array}{c}
\boldsymbol{\theta}_{1}(\omega) \\
\boldsymbol{\theta}_{2}(\omega)
\end{array}\right]=\left[\begin{array}{c}
\eta(\omega) \mathcal{F}(\omega) \\
0
\end{array}\right],
$$

in which $\mathcal{F}(\omega) \in \mathbb{C}^{N_{1}^{S}}$ is written as

$$
\mathcal{F}(\omega)=\left[P_{1}^{S}\right]^{T} \mathbf{F}(\omega)
$$

and where

$$
\begin{gathered}
{\left[\mathcal{A}_{1}(\omega)\right]=\left[P_{1}^{S}\right]^{T}\left[A_{1}(\omega)\right]\left[P_{1}^{S}\right] \quad, \quad\left[\mathcal{A}_{2}(\omega)\right]=\left[P_{2}\right]^{T}\left[A_{2}(\omega)\right]\left[P_{2}\right],} \\
{[\mathcal{J}]=\left[P_{1}^{S}\right]^{T}[\boldsymbol{J}]\left[P_{1}^{S}\right]=\left(\left[P_{1}^{S}\right]^{T} \boldsymbol{\Pi}_{2}\right)\left(\left[P_{1}^{S}\right]^{T} \boldsymbol{\Pi}_{2}\right)^{T},} \\
{[\mathcal{C}]=\left[P_{1}^{S}\right]^{T}[C]\left[P_{2}\right] .}
\end{gathered}
$$

From Eqs. (27) and (44), we deduce that for all $\mathbf{x}$ in $\Omega_{1}$, the displacement field of the structure is written as

$$
\widetilde{\mathbf{u}}(\mathbf{x}, \omega)=\sum_{k=1}^{N_{1}^{S}} \mathcal{U}_{k}(\mathbf{x}) \theta_{1, k}(\omega)
$$

in which $\mathcal{U}_{k}(\mathbf{x}) \in \mathbb{C}^{3}$ is written as

$$
\mathcal{U}_{k}(\mathbf{x})=\sum_{\alpha=1}^{n_{1}}\left[P_{1}^{S}\right]_{\alpha k} \mathbf{u}_{\alpha}(\mathbf{x})
$$

From Eqs. (35), (44) and (45), we deduce that for all $\mathbf{x}$ in $\Omega_{2}$, the pressure field in the internal acoustic fluid is written as

$$
\widetilde{p}(\mathbf{x}, \omega)=\sum_{k=1}^{N_{1}^{S}} \mathcal{P}_{k}(\mathbf{x}, \omega) \theta_{1, k}(\omega)
$$

J. Acoust. Soc. Am., 106(6), 3362-3374 (1999). 
in which $\mathcal{P}(\mathbf{x}, \omega)=\left(\mathcal{P}_{1}(\mathbf{x}, \omega), \ldots, \mathcal{P}_{N_{1}^{S}}(\mathbf{x}, \omega)\right) \in \mathbb{C}^{N_{1}^{S}}$ is such that

$$
\mathcal{P}(\mathbf{x}, \omega)=\omega^{2} \rho_{2}[\mathcal{C}]\left[\mathcal{A}_{2}(\omega)\right]^{-1}\left[P_{2}\right]^{T} \Psi(\mathbf{x})-\kappa\left[P_{1}^{S}\right]^{T} \Pi_{2}
$$

Equations (45), (50) and (52) constitute the reduced model adapted to MF band $B$.

\section{CONSTRUCTION OF DOMINANT EIGENSUBSPACES}

Concerning the construction of the dominant eigensubspace of the energy operator relative to MF band $B$ for the structure on the one hand, and for the internal acoustic fluid on the other hand, we can use the indirect procedure in the frequency domain or the procedure based on the use of the MF solving method in the time domain presented in Refs. 1 and 2. A detailed analysis of these procedures cannot be reproduced here. Nevertheless, in order to facilitate the understanding of Sections VII and VIII, we summarize below the main results of the MF solving method in the time domain that we use for the examples. For $a=1$ and $a=2$, the problem defined by Eqs. (36) to (38) is solved by calculating the $N_{a}$ lowest eigenvalues of the following generalized symmetric eigenvalue problem

$$
\begin{gathered}
{\left[G_{a}\right]\left[S_{a}\right]=\left[H_{a}\right]\left[S_{a}\right]\left[\Gamma_{a}\right],} \\
{\left[S_{a}\right]^{T}\left[H_{a}\right]\left[S_{a}\right]=\left[I_{a}\right],} \\
{\left[S_{a}\right]^{T}\left[G_{a}\right]\left[S_{a}\right]=\left[\Gamma_{a}\right],}
\end{gathered}
$$

for which the subspace iteration algorithm ${ }^{1,12-15}$ is used. The dimension $m_{a}$ of the subspace used for iterations is such that $N_{a}<m_{a} \ll n_{a}$ with $m_{a}=\left\{2 N_{a}, N_{a}+8\right\}$. Consequently, $\left[S_{a}\right]$ is an $\left(n_{a} \times m_{a}\right)$ real matrix and $\left[\Gamma_{a}\right]$ is a diagonal $\left(m_{a} \times m_{a}\right)$ real matrix. We have

$$
\begin{gathered}
{\left[\widetilde{\Lambda}_{a}\right]=\left[\Gamma_{a}\right]^{-1},} \\
{\left[\widetilde{P}_{a}\right]=\left[S_{a}\right]\left[\Gamma_{a}\right]^{-1 / 2},}
\end{gathered}
$$

where $\left[\widetilde{P}_{a}\right]$ is the $\left(n_{a} \times m_{a}\right)$ real matrix whose first $N_{a}$ columns are eigenvectors $\mathbf{P}_{a}^{1}, \ldots, \mathbf{P}_{a}^{N_{a}}$ defining matrix $\left[P_{a}\right]$. For each iteration of the subspace iteration algorithm, we only need to calculate an $\left(n_{a} \times m_{a}\right)$ real matrix $\left[W_{a}\right]=\left[E_{a}\right]\left[X_{a}\right]$, in which $\left[X_{a}\right]$ is a given $\left(n_{a} \times m_{a}\right)$ real matrix. Let $\chi_{0}(t)$ be the complex-valued function defined on $\mathbb{R}$ by $\chi_{0}(t)=e^{-i \omega_{B} t} \chi(t)$ in which $\chi(t)=(1 / 2 \pi) \int_{B} e^{i \omega t} \widehat{\chi}(\omega) d \omega$ with

$$
\widehat{\chi}(\omega)=\frac{1}{\pi} \omega^{2}|\eta(\omega)|^{2} \mathbb{1}_{B}(\omega)
$$

Therefore, $\chi_{0}$ is an LF signal whose band is $[-\Delta \omega / 2, \Delta \omega / 2]$. Then it is proved ${ }^{1}$ that $\left[W_{a}\right]$ can be calculated by

$$
\left[W_{a}\right]=2 \pi \Re e\left\{\left[Z_{a}(0)\right]\right\} \quad,
$$

in which $\left[Z_{a}(t)\right]$ is the solution of the following LF equations in the time domain associated with the MF equations,

$$
\begin{aligned}
{\left[M_{a}\right]\left[\ddot{Y}_{a}(t)\right]+\left[\widetilde{D}_{a}\right]\left[\dot{Y}_{a}(t)\right]+\left[\widetilde{K}_{a}\right]\left[Y_{a}(t)\right] } & \left.=\chi_{0}(t)\left[X_{a}\right], t \in\right]-\infty,+\infty[, \\
{\left[M_{a}\right]\left[\ddot{Z}_{a}(t)\right]+\left[\widetilde{D}_{a}\right]\left[\dot{Z}_{a}(t)\right]+\left[\widetilde{K}_{a}\right]\left[Z_{a}(t)\right] } & \left.=\left[M_{a}\right] \overline{\left[Y_{a}(-t)\right]}, t \in\right]-\infty, 0[
\end{aligned}
$$


in which symmetric $\left(n_{a} \times n_{a}\right)$ complex matrices $\left[\widetilde{D}_{a}\right]$ and $\left[\widetilde{K}_{a}\right]$ are written, for $a=1$ and $a=2$, as

$$
\begin{gathered}
{\left[\widetilde{D}_{a}\right]=\left[D_{a}\left(\omega_{B}\right)\right]+2 i \omega_{B}\left[M_{a}\right],} \\
{\left[\widetilde{K}_{a}\right]=-\omega_{B}^{2}\left[M_{a}\right]+i \omega_{B}\left[D_{a}\left(\omega_{B}\right)\right]+\left[K_{a}\left(\omega_{B}\right)\right] .}
\end{gathered}
$$

It should be noted that for $a=2,\left[K_{2}\left(\omega_{B}\right)\right]=\left[K_{2}\right]$. In addition, if the constraint $\int_{\Omega_{2}} \psi d \mathbf{x}=0$ is not included in the discretization, then, for $a=2$, Eqs. (61) and (62) must be solved with the constraint (see Section II)

$$
\mathbf{L}^{T}\left[Y_{2}(t)\right]=[0] \quad ; \quad \mathbf{L}^{T}\left[Z_{2}(t)\right]=[0] .
$$

The LF Eqs. (61) and (62) are solved using an unconditionally stable implicit step-by-step integration method. Concerning Eq. (61), time interval $]-\infty, \infty[$ is replaced by the finite interval $\left.] t_{I}, t_{F}\right]$ with the initial conditions $\left[Y_{a}\left(t_{I}\right)\right]=\left[\dot{Y}_{a}\left(t_{I}\right)\right]=[0]$. Concerning Eq. (62), time interval $]-\infty, 0]$ is replaced by the finite interval $\left.]-t_{F}, 0\right]$ with the initial conditions $\left[Z_{a}\left(-t_{F}\right)\right]=\left[\dot{Z}_{a}\left(-t_{F}\right)\right]=[0]$.

\section{TIME-STATIONARY RANDOM RESPONSE USING THE REDUCED MODEL}

The structural-acoustic system is submitted to a time-stationary second-order centered random wall pressure field $\left\{p(\mathbf{x}, t), \mathbf{x} \in \Gamma_{1}, t \in \mathbb{R}\right\}$ with values in $\mathbb{R}$ and we are interested in the stationary response of the structural-acoustic system. The cross-correlation function of random field $p$ is denoted as $R_{p}(\mathbf{x}, \mathbf{y}, \tau)=\mathbb{E}\{p(\mathbf{x}, t+\tau) p(\mathbf{y}, t)\}$ in which $\mathbb{E}$ is the mathematical expectation and is such that ${ }^{16}$

$$
R_{p}(\mathbf{x}, \mathbf{y}, \tau)=\int_{\mathbb{R}} e^{i \omega \tau} S_{p}(\mathbf{x}, \mathbf{y}, \omega) d \omega
$$

in which $S_{p}(\mathbf{x}, \mathbf{y}, \omega)$ is the cross-spectral density function which is written as

$$
S_{p}(\mathbf{x}, \mathbf{y}, \omega)=|\eta(\omega)|^{2} s_{p}(\mathbf{x}, \mathbf{y}, \omega)
$$

Let $\left\{\mathbb{F}_{\alpha}(t), t \in \mathbb{R}\right\}$ be the stochastic process defined by

$$
\mathbb{F}_{\alpha}(t)=-\int_{\Gamma_{1}} p(\mathbf{x}, t) \mathbf{n}_{1}(\mathbf{x}) \cdot \mathbf{u}_{\alpha}(\mathbf{x}) d s(\mathbf{x})
$$

Therefore the $\left(n_{1} \times n_{1}\right)$ matrix-valued spectral density function $\left[S_{\mathbb{F}}(\omega)\right]$ of stationary stochastic process $\mathbb{F}=\left(\mathbb{F}_{1}, \ldots, \mathbb{F}_{n_{1}}\right)$ indexed by $\mathbb{R}$ with values in $\mathbb{R}^{n_{1}}$ is such that

$$
\left[S_{\mathbb{F}}(\omega)\right]_{\alpha \alpha^{\prime}}=\int_{\Gamma_{1}} \int_{\Gamma_{1}}|\eta(\omega)|^{2} s_{p}(\mathbf{x}, \mathbf{y}, \omega)\left\{\mathbf{n}_{1}(\mathbf{x}) \cdot \mathbf{u}_{\alpha}(\mathbf{x})\right\} \times\left\{\mathbf{n}_{1}(\mathbf{y}) \cdot \mathbf{u}_{\alpha^{\prime}}(\mathbf{y})\right\} d s(\mathbf{x}) d s(\mathbf{y})
$$

From Eq. (50), we deduce that, for all $\mathbf{x}$ and $\mathbf{y}$ fixed in $\Omega_{1}$, the $(3 \times 3)$ matrix-valued cross-spectral density function $\left[S_{\tilde{\mathbf{u}}}(\mathbf{x}, \mathbf{y}, \omega)\right]$ of the $\mathbb{R}^{3}$-valued stochastic field $\left\{\widetilde{\mathbf{u}}(\mathbf{x}, t), \mathbf{x} \in \Omega_{1}, t \in\right.$ $\mathbb{R}\}$, such that

$$
\left[R_{\tilde{\mathbf{u}}}(\mathbf{x}, \mathbf{y}, \tau)\right]=\mathbb{E}\left\{\widetilde{\mathbf{u}}(\mathbf{x}, t+\tau) \widetilde{\mathbf{u}}(\mathbf{y}, t)^{T}\right\}=\int_{\mathbb{R}} e^{i \omega \tau}\left[S_{\tilde{\mathbf{u}}}(\mathbf{x}, \mathbf{y}, \omega)\right] d \omega
$$

J. Acoust. Soc. Am., 106(6), 3362-3374 (1999). 
can be written as

$$
\left[S_{\tilde{\mathbf{u}}}(\mathbf{x}, \mathbf{y}, \omega)\right]=\sum_{j=1}^{N_{1}^{S}} \sum_{k=1}^{N_{1}^{S}}\left[S_{\boldsymbol{\theta}_{1}}(\omega)\right]_{j k} \mathcal{U}_{j}(\mathbf{x}) \mathcal{U}_{k}(\mathbf{y})^{T}
$$

in which $\left[S_{\boldsymbol{\theta}_{1}}(\omega)\right]$ is the matrix-valued spectral density function of $\mathbb{R}^{N_{1}^{S}}$-valued mean-square stationary stochastic process $\left\{\boldsymbol{\theta}_{1}(t), t \in \mathbb{R}\right\}$. From Eq. (52), we deduce that, for all $\mathbf{x}$ and $\mathbf{y}$ fixed in $\Omega_{2}$, the complex-valued cross-spectral density function $S_{\tilde{p}}(\mathbf{x}, \mathbf{y}, \omega)$ of the real-valued stochastic field $\left\{\widetilde{p}(\mathbf{x}, t), \mathbf{x} \in \Omega_{2}, t \in \mathbb{R}\right\}$, such that

$$
R_{\tilde{p}}(\mathbf{x}, \mathbf{y}, \tau)=\mathbb{E}\{\widetilde{p}(\mathbf{x}, t+\tau) \widetilde{p}(\mathbf{y}, t)\}=\int_{\mathbb{R}} e^{i \omega \tau} S_{\tilde{p}}(\mathbf{x}, \mathbf{y}, \omega) d \omega
$$

can be written as

$$
S_{\tilde{p}}(\mathbf{x}, \mathbf{y}, \omega)=\sum_{j=1}^{N_{1}^{S}} \sum_{k=1}^{N_{1}^{S}}\left[S_{\boldsymbol{\theta}_{1}}(\omega)\right]_{j k} \mathcal{P}_{j}(\mathbf{x}, \omega) \overline{\mathcal{P}_{k}(\mathbf{y}, \omega)}
$$

From Eqs. (45) and (46), and using linear filtering of stationary stochastic processes ${ }^{16-18}$, we deduce that matrix-valued spectral density function $\left[S_{\boldsymbol{\theta}_{1}}(\omega)\right]$ (appearing in Eqs. (71) and (73)) is written as

$$
\left[S_{\boldsymbol{\theta}_{1}}(\omega)\right]=[\mathcal{T}(\omega)]\left[P_{1}^{S}\right]^{T}\left[S_{\mathbb{F}}(\omega)\right]\left[P_{1}^{S}\right][\mathcal{T}(\omega)]^{*},
$$

in which $\left(N_{1}^{S} \times N_{1}^{S}\right)$ symmetric complex matrix $[\mathcal{T}(\omega)]$ is written as

$$
[\mathcal{T}(\omega)]=\left(\left[\mathcal{A}_{1}(\omega)\right]+\kappa[\mathcal{J}]-\omega^{2}[\mathcal{C}]\left[\mathcal{A}_{2}(\omega)\right]^{-1}[\mathcal{C}]^{T}\right)^{-1}
$$

\section{VALIDATION FOR A STRUCTURE HAVING AN MF BEHAVIOR COUPLED WITH AN INTERNAL ACOUSTIC FLUID HAVING AN LF BEHAVIOR IN MF BAND $B$}

This first example concerns an inhomogeneous structure having an MF behavior in a given narrow MF band $B$, coupled with an internal acoustic cavity filled with a gas and having an LF behavior in MF band $B$. We consider the MF time-stationary random response of this structural-acoustic system in MF band $B$, the structure being excited by a time-stationary random wall pressure field.

\section{A. Description of the structural-acoustic system}

The structural-acoustic system is referenced to an $\left(x_{1}, x_{2}, x_{3}\right)$ coordinate system. The structure is a rectangular thin plate in bending mode, located in plane $O x_{1} x_{2}$, to which are attached two point masses, three springs and five dashpots, as shown in Figure 2. Domain $\Omega_{1}=\Gamma_{1}$ of the plate (middle surface) is rectangular and the plate is simply supported, homogeneous and isotropic, with constant thickness, width $L_{1}=0.5 \mathrm{~m}$, length $L_{2}=1.0 \mathrm{~m}$, surfacemass density $\rho_{1}=40 \mathrm{~kg} / \mathrm{m}^{2}$, total mass $\mu_{1}=\rho_{1} L_{1} L_{2}=20 \mathrm{~kg}$ and constant damping rate $\xi_{1}=0.002$. We assume that the usual thin plate theory can be used. The thickness, 
Young's modulus and Poisson's ratio of the plate are such that the lowest eigenfrequency of the associated conservative plate (without point masses and springs) is $5 \mathrm{~Hz}$. To this plate are attached (1) two point masses having a mass of $3 \mathrm{~kg}$ and $4 \mathrm{~kg}$ located at points $(0.2,0.4,0)$ and $(0.35,0.75,0)$ respectively, (2) three springs having the same stiffness coefficient $k=\varepsilon_{k} \mu_{1} \omega_{r e f}^{2}$ with $\omega_{\text {ref }}=2 \pi \times 550 \mathrm{rad} / \mathrm{s}, \varepsilon_{k}=0.1$, located at points $(0.221,0.278,0)$, $(0.332,0.537,0)$ and $(0.443,0.826,0)$, and (3) five dashpots having the same damping coefficient $d=2 \varepsilon_{d} \mu_{1} \xi_{1} \omega_{\text {ref }}$ with $\varepsilon_{d}=0.1$, located at points $(0.154,0.165,0),(0.145,0.334,0)$, $(0.465,0.373,0),(0.247,0.462,0)$ and $(0.268,0.681,0)$. This structure (the plate with point masses, springs and dashpots) is coupled with an acoustic cavity constituted of a rectangular room $\Omega_{2}$ (see Figure 2) bounded by five rigid walls lying along the planes $x_{1}=0, x_{1}=L_{1}$, $x_{2}=0, x_{2}=L_{2}, x_{3}=L_{3}$ with $L_{3}=0.318 \mathrm{~m}$. The sixth wall lying in plane $x_{3}=0$ is not rigid and is constituted by the plate. This bounded room is filled with a gas having a constant mass density $\rho_{2}=1 \mathrm{~kg} / \mathrm{m}^{3}$, speed of sound $c_{2}=330 \mathrm{~m} / \mathrm{s}$ and dissipative coefficient $\tau=\xi_{2} / \omega_{\text {ref }}$ with $\xi_{2}=0.002$. The total mass of the gas is $\mu_{2}=\rho_{2} L_{1} L_{1} L_{3}=0.159$ $\mathrm{kg}$. We consider the MF response of this structural-acoustic system in narrow MF band $B=2 \pi \times[500,550] \mathrm{rad} / \mathrm{s}$, i.e. on the $[500,550] \mathrm{Hz}$ frequency band. The excitation is time-stationary random wall pressure field $\left\{p(\mathbf{x}, t), \mathbf{x} \in \Gamma_{1}, t \in \mathbb{R}\right\}$ introduced in Section VI, for which the cross-spectral density function defined by Eq. (67) is such that for all $\omega$ in $B$, $|\eta(\omega)|=1$ and $s_{p}(\mathbf{x}, \mathbf{y}, \omega)=\left(L_{1} L_{2}\right)^{-1} \delta_{0}\left(x_{1}-y_{1}\right) \delta_{0}\left(x_{2}-y_{2}\right)$ where $\delta_{0}$ is the Dirac delta function on $\mathbb{R}$ at point 0 . For the validation, we are interested in calculating the power spectral density function $S_{\text {struc }}(\omega)$ relative to the structure and defined by

$$
S_{\text {struc }}(\omega)=\frac{1}{|\eta(\omega)|^{2}\left|\Omega_{1}\right|} \int_{\Omega_{1}} \operatorname{tr}\left[S_{\tilde{\mathbf{u}}}(\mathbf{x}, \mathbf{x}, \omega)\right] d \mathbf{x}
$$

and the power spectral density function $S_{\text {acous }}(\omega)$ relative to the acoustic cavity and defined by

$$
S_{\text {acous }}(\omega)=\frac{1}{\rho_{2}^{2} c_{2}^{4}} \frac{1}{|\eta(\omega)|^{2}\left|\Omega_{2}\right|} \int_{\Omega_{2}}\left[S_{\tilde{p}}(\mathbf{x}, \mathbf{x}, \omega)\right] d \mathbf{x}
$$

\section{B. Description of the finite dimension approximation}

The finite dimension approximation is defined in Section II. Concerning the structure in vacuo (plate with point masses, springs and dashpots), family $\mathbf{u}_{1}, \ldots, \mathbf{u}_{n_{1}}$ is chosen as the first $n_{1}$ modes of the associated conservative plate (without point masses and springs) whose corresponding eigenfrequencies are $f_{\text {plate }, 1} \leq f_{\text {plate }, 2} \leq \ldots \leq f_{\text {plate }, n_{1}}$. For $\alpha=\left(\alpha_{1}, \alpha_{2}\right)$, $\alpha_{1} \geq 1$ and $\alpha_{2} \geq 1$, we have $\mathbf{u}_{\alpha}(\mathbf{x})=\left(u_{\alpha, 1}(\mathbf{x}), u_{\alpha, 2}(\mathbf{x}), u_{\alpha, 3}(\mathbf{x})\right)$ with $u_{\alpha, 1}(\mathbf{x})=u_{\alpha, 2}(\mathbf{x})=$ 0 and

$$
u_{\alpha, 3}(\mathbf{x})=2 \sin \left(\frac{\alpha_{1} \pi x_{1}}{L_{1}}\right) \sin \left(\frac{\alpha_{2} \pi x_{2}}{L_{2}}\right) .
$$

From a convergence study of the structural-acoustic response over the $[0,700] \mathrm{Hz}$ frequency band, we deduced the value of $n_{1}$ which is $n_{1}=407$. We have $f_{\text {plate }, 1}=5 \mathrm{~Hz}$ and $f_{\text {plate }, 407}=1097 \mathrm{~Hz}$. For all $\alpha$, the normalization of $\mathbf{u}_{\alpha}$ is such that

$$
\int_{0}^{L_{1}} \int_{0}^{L_{2}} \rho_{1}\left|u_{\alpha, 3}\left(x_{1}, x_{2}\right)\right|^{2} d x_{1} d x_{2}=\mu_{1} .
$$

The $\left(n_{1} \times n_{1}\right)$ matrices $\left[M_{1}\right],\left[D_{1}\right]$ and $\left[K_{1}\right]$ are independent of the frequency and are dense due to the presence of the point masses, dashpots and springs. Matrix $\left[G_{1}\right]=L_{1} L_{2}\left[I_{1}\right]$ is 
diagonal, $\left[I_{1}\right]$ being the $\left(n_{1} \times n_{1}\right)$ unity matrix. The lowest eigenfrequency of the associated conservative structure in vacuo (plate with point masses and springs) is $f_{1,1}=7.74 \mathrm{~Hz}$ (to be compared with $f_{\text {plate }, 1}=5 \mathrm{~Hz}$ ). There are 255 modes of the structure in vacuo in frequency band $[0,700] \mathrm{Hz}, 179$ structural modes in frequency band $[0,500] \mathrm{Hz}$ and 20 structural modes in narrow MF band $[500,550] \mathrm{Hz}$. Then the rank of the first structural mode belonging to frequency band $[500,550] \mathrm{Hz}$ is 180 and consequently, the structure has an MF behavior in frequency band $B$.

Concerning the acoustic cavity, family $\psi_{1}, \ldots, \psi_{n_{2}}$ is chosen as the first $n_{2}$ acoustic modes of the acoustic cavity with rigid walls whose corresponding eigenfrequencies are $f_{2,1} \leq f_{2,2} \leq$ $\ldots \leq f_{2, n_{2}}$. For $\beta=\left(\beta_{1}, \beta_{2}, \beta_{3}\right)$ with $\beta_{1}+\beta_{2}+\beta_{3} \neq 0$ and $\beta_{1}, \beta_{2}, \beta_{3} \geq 0$, we have

$$
\psi_{\beta}(\mathbf{x})=2 \sqrt{2} \cos \left(\frac{\beta_{1} \pi x_{1}}{L_{1}}\right) \cos \left(\frac{\beta_{2} \pi x_{2}}{L_{2}}\right) \cos \left(\frac{\beta_{3} \pi x_{3}}{L_{3}}\right)
$$

These functions satisfy the constraint $\int_{\Omega_{2}} \psi_{\beta}(\mathbf{x}, \omega) d \mathbf{x}=0$. From a convergence study of the structural-acoustic response over the $[0,700] \mathrm{Hz}$ frequency band, we deduced the value of $n_{2}$ which is $n_{2}=19$. For all $\beta$, the normalization of $\psi_{\beta}$ is such that

$$
\int_{0}^{L_{1}} \int_{0}^{L_{2}} \int_{0}^{L_{3}} \rho_{2}\left|\psi_{\beta}(\mathbf{x})\right|^{2} d \mathbf{x}=\mu_{2}
$$

The $\left(n_{2} \times n_{2}\right)$ matrices $\left[M_{2}\right],\left[D_{2}\right]$ and $\left[K_{2}\right]$ are independent of the frequency and are diagonal. Matrix $\left[G_{2}\right]=L_{1} L_{2} L_{3}\left[I_{2}\right]$ is diagonal, $\left[I_{2}\right]$ being the $\left(n_{2} \times n_{2}\right)$ unity matrix. The lowest acoustic eigenfrequency of the acoustic cavity with rigid walls is $f_{2,1}=165 \mathrm{~Hz}$. There are 16 acoustic modes in frequency band $[0,700] \mathrm{Hz}, 6$ acoustic modes in frequency band $[0,500]$ $\mathrm{Hz}$ and 2 acoustic modes in narrow MF band $[500,550] \mathrm{Hz}$. Then the rank of the first acoustic mode in MF band $[500,550] \mathrm{Hz}$ is 7 and consequently, the acoustic cavity has an LF behavior in MF band $B$.

For $\alpha=\left(\alpha_{1}, \alpha_{2}\right)$, component $\Pi_{2, \alpha}$ of vector $\Pi_{2} \in \mathbb{R}^{n_{1}}$, defined by Eq. (32), is such that

$$
\Pi_{2, \alpha}=-\frac{2 L_{1} L_{2}}{\alpha_{1} \alpha_{2} \pi^{2}} \times\left\{\cos \left(\alpha_{1} \pi\right)-1\right\} \times\left\{\cos \left(\alpha_{2} \pi\right)-1\right\} .
$$

For $\alpha=\left(\alpha_{1}, \alpha_{2}\right)$ and $\beta=\left(\beta_{1}, \beta_{2}, \beta_{3}\right)$, coupling $\left(n_{1} \times n_{2}\right)$ real matrix $[C]$, defined by Eq. (34), is such that

$$
\begin{aligned}
{[C]_{\alpha \beta}=-\frac{\sqrt{2} \rho_{2} L_{1} L_{2}}{\pi^{2}} } & \times\left\{\frac{1-\cos \left\{\pi\left(\alpha_{1}+\beta_{1}\right)\right\}}{\alpha_{1}+\beta_{1}}+\frac{1-\cos \left\{\pi\left(\alpha_{1}-\beta_{1}\right)\right\}}{\alpha_{1}-\beta_{1}}\right\} \\
& \times\left\{\frac{1-\cos \left\{\pi\left(\alpha_{2}+\beta_{2}\right)\right\}}{\alpha_{2}+\beta_{2}}+\frac{1-\cos \left\{\pi\left(\alpha_{2}-\beta_{2}\right)\right\}}{\alpha_{2}-\beta_{2}}\right\} .
\end{aligned}
$$

Finally, from Eq. (69), we deduce that $\left[S_{\mathbb{F}}(\omega)\right]_{\alpha \alpha^{\prime}}=|\eta(\omega)|^{2} \delta_{\alpha \alpha^{\prime}}$.

\section{Constructing the reference solution on a broad frequency band}

The reference solution is constructed on the $[5,700] \mathrm{Hz}$ broad frequency band with a sampling frequency step $\delta \nu=0.165 \mathrm{~Hz}$. The power spectral density functions $S_{\text {struc }}^{\text {ref }}(\omega)$ and $S_{\text {acous }}^{\text {ref }}(\omega)$ corresponding to the reference solution and defined by Eqs. (76) and (77) are directly 
calculated in the frequency domain without using the reduced model. From Eqs. (76), (77), (27), (29) and (35), we deduce that

$$
\begin{array}{r}
S_{\text {struc }}^{\text {ref }}(\omega)=\operatorname{tr}\left\{[T(\omega)][T(\omega)]^{*}\right\}, \\
S_{\text {acous }}^{\text {ref }}(\omega)=\frac{1}{\rho_{2}^{2} c_{2}^{4}}\left(\operatorname{tr}\left\{([V(\omega)][T(\omega)])([V(\omega)][T(\omega)])^{*}\right\}\right. \\
\left.+\kappa^{2}\left([T(\omega)] \Pi_{2}\right)^{*}\left([T(\omega)] \Pi_{2}\right)\right),
\end{array}
$$

in which $[T(\omega)]$ is the symmetric $\left(n_{1} \times n_{1}\right)$ complex matrix which is written as

$$
[T(\omega)]=\left(\left[A_{1}(\omega)\right]+\kappa[J]-\omega^{2}[C]\left[A_{2}(\omega)\right]^{-1}[C]^{T}\right)^{-1}
$$

where $\left[A_{1}(\omega)\right],[J],\left[A_{2}(\omega)\right]$ and $[C]$ are the matrices defined by Eqs. (30), (32) with (78), (33) and (79) respectively. In Eq. (81), $\left(n_{2} \times n_{1}\right)$ complex matrix $[V(\omega)]$ is defined by

$$
[V(\omega)]=\omega^{2} \rho_{2}\left[A_{2}(\omega)\right]^{-1}[C]^{T}
$$

Figs. 3 and 4 show the mappings $\nu \mapsto 10 \times \log _{10}\left(S_{\text {struc }}^{\text {ref }}(2 \pi \nu)\right)$ and $\nu \mapsto 10 \times \log _{10}\left(S_{\text {acous }}^{\text {ref }}(2 \pi \nu)\right)$ respectively over the $[5,700] \mathrm{Hz}$ broad frequency band.

\section{Reference solution on the narrow MF band}

The reference solution on narrow MF band $B=[500,550] \mathrm{Hz}$ is constructed as explained in Section VII C using the frequency resolution $\delta \nu=0.166 \mathrm{~Hz}$. The graphs of functions $\nu \mapsto 10 \times \log _{10}\left(S_{\text {struc }}^{\text {ref }}(2 \pi \nu)\right)$ and $\nu \mapsto 10 \times \log _{10}\left(S_{\text {acous }}^{\text {ref }}(2 \pi \nu)\right)$ (corresponding to Figures 3 and 4) of the reference solution on narrow MF band $B$ are used below (see the solid lines in Figures 7 to 10) to evaluate the accuracy of the response constructed using the reduced model.

\section{E. Constructing the dominant eigensubspaces}

For the structure, the dominant eigensubspace of the energy operator relative to band $B$ for the structure in vacuo (related to matrix $\left[E_{1}\right]$ defined by Eqs. (41) to (43)) is constructed using the method presented in Section $\mathrm{V}$ with $N_{1}=50$. Figure 5 shows the graph of the function $j \mapsto 10 \times \log _{10}\left(\lambda_{1}^{j}\right)$ for $j \in\{1,2, \ldots, 50\}$ in which $\lambda_{1}^{1}, \ldots, \lambda_{1}^{50}$ are the highest eigenvalues of the generalized symmetric eigenvalue problem defined by Eq. (36) for $a=1$. There is a strong decrease in the eigenvalues which means there exists the possibility of constructing an efficient reduced model for the structure. Figure 5 shows that the order $N_{1}$ of the reduced model is about 25 for band $B$.

For the internal acoustic fluid, the dominant eigensubspace of the energy operator relative to band $B$ for the internal acoustic cavity with rigid walls (related to matrix $\left[E_{2}\right]$ defined by Eqs. (41) to (43)) is constructed using the method presented in Section V with $N_{2}=17$. Figure 6 shows the graph of the function $j \mapsto 10 \times \log _{10}\left(\lambda_{2}^{j}\right)$ for $j \in\{1,2, \ldots, 17\}$ in which $\lambda_{2}^{1}, \ldots, \lambda_{2}^{17}$ are the highest eigenvalues of the generalized symmetric eigenvalue problem defined by Eq. (36) for $a=2$. Figure 6 shows that the order $N_{2}$ of the reduced model is about 4 for band $B$.

\section{F. Reduced model adapted to the narrow MF band}

In this section, we present a comparison of the reference solution constructed in Section VII D with the solution obtained by the reduced model constructed using the results of Sections IV 
and VI and Eqs. (76) and (77). Two reduced models are considered below. For the first reduced model, the parameters are $N_{1}^{S}=N_{1}=25$ (then $N_{0}=0$ ) and $N_{2}=4$. Figure 7 is related to the structure and shows the comparison between function $\nu \mapsto 10 \times \log _{10}\left(S_{\text {struc }}^{\text {ref }}(2 \pi \nu)\right)$ (solid line corresponding to the reference solution) and function $\nu \mapsto 10 \times \log _{10}\left(S_{\text {struc }}(2 \pi \nu)\right.$ ) (dashed line corresponding to the first reduced model) on narrow MF band [500, 550] Hz. Figure 8 is related to the internal acoustic fluid and shows the comparison between function $\nu \mapsto 10 \times \log _{10}\left(S_{\text {acous }}^{\text {ref }}(2 \pi \nu)\right)$ (solid line corresponding to the reference solution) and function $\nu \mapsto 10 \times \log _{10}\left(S_{\text {acous }}(2 \pi \nu)\right.$ ) (dashed line corresponding to the first reduced model) on narrow MF band $[500,550] \mathrm{Hz}$. Figure 7 shows that the comparison is very good for the structure, but Figure 8 shows that this first reduced model $\left(N_{0}=0\right)$ is not so good for the internal acoustic fluid. The problem which appears in Figure 8 has been explained in Sections III C and III D; to accelerate convergence of the reduced model for prediction of the acoustic pressure in the internal acoustic fluid, it is necessary to take $N_{0}>0$ (see below).

For the second reduced model, the parameters are $N_{1}=25, N_{0}=10$ (then $N_{1}^{S}=35$ ) and $N_{2}=4$. Figure 9 is related to the structure and shows the comparison between function $\nu \mapsto 10 \times \log _{10}\left(S_{\text {struc }}^{\text {ref }}(2 \pi \nu)\right)$ (solid line corresponding to the reference solution) and function $\nu \mapsto 10 \times \log _{10}\left(S_{\text {struc }}(2 \pi \nu)\right)$ (dashed line corresponding to the second reduced model) on narrow MF band $[500,550] \mathrm{Hz}$. Figure 10 is related to the internal acoustic fluid and shows the comparison between function $\nu \mapsto 10 \times \log _{10}\left(S_{\text {acous }}^{\text {ref }}(2 \pi \nu)\right)$ (solid line corresponding to the reference solution) and function $\nu \mapsto 10 \times \log _{10}\left(S_{\text {acous }}(2 \pi \nu)\right)$ (dashed line corresponding to the second reduced model) on narrow MF band $[500,550] \mathrm{Hz}$. Figure 9 is quite similar to Figure 7 and shows that the comparison is very good for the structure and Figure 10 shows that this second reduced model $\left(N_{0}=10\right)$ is good. Consequently, for this structural-acoustic system whose structure has an MF behavior and whose internal acoustic fluid has an LF behavior (MF-LF coupling), the reduced model developed in Section IV based on the use of the structural vector basis constructed in Section III D is validated.

\section{VALIDATION FOR A STRUCTURE HAVING AN MF BEHAVIOR COUPLED WITH AN INTERNAL ACOUSTIC FLUID HAVING AN MF BEHAVIOR IN MF BAND $B$}

This second example concerns the inhomogeneous structure defined in Section VII, having an MF behavior in given narrow MF band $B$, coupled with an internal acoustic cavity filled with a gas and having an MF behavior in MF band $B$. We consider the MF time-stationary random response of this structural-acoustic system in MF band $B$, the structure being excited by the time-stationary random wall pressure field used in Section VII.

\section{A. Description of the structural-acoustic system}

We consider the structural-acoustic system defined in Section VII A for which acoustic rectangular room $\Omega_{2}$ is such that $L_{3}=9.0 \mathrm{~m}$ (instead of $L_{3}=0.318 \mathrm{~m}$, see Figure 2). The total mass of the gas is $\mu_{2}=\rho_{2} L_{1} L_{1} L_{3}=4.5 \mathrm{~kg}$. We consider the MF response of this structural-acoustic system in narrow MF band $B=[500,550] \mathrm{Hz}$. The excitation is the time-stationary random wall pressure field defined in Section VII A. For the validation, we are interested in calculating the power spectral density functions defined by Eqs. (76) and (77).

\section{B. Description of the finite dimension approximation}

The finite dimension approximation of the structure in vacuo (plate with point masses, springs and dashpots) is defined in Section VII B. We have $n_{1}=407$. There are 255 modes of the 
structure in vacuo in frequency band $[0,700] \mathrm{Hz}, 179$ structural modes in frequency band $[0,500] \mathrm{Hz}$ and 20 structural modes in narrow MF band $[500,550] \mathrm{Hz}$. Then the rank of the first structural mode belonging to frequency band $[500,550] \mathrm{Hz}$ is 180 and consequently, the structure has an MF behavior in frequency band $B$.

Concerning the acoustic cavity, we use the finite dimension approximation defined in Section VII $\mathrm{B}$ for which the value of $n_{2}$ has to be increased. From a convergence study of the structural-acoustic response over the $[0,700] \mathrm{Hz}$ frequency band, we deduced the value of $n_{2}$ which is $n_{2}=353$ (instead of 19). The lowest acoustic eigenfrequency of the acoustic cavity with rigid walls is $f_{2,1}=18.33 \mathrm{~Hz}$. There are 289 acoustic modes in frequency band $[0,700]$ $\mathrm{Hz}, 128$ acoustic modes in frequency band $[0,500] \mathrm{Hz}$ and 34 acoustic modes in narrow MF band $[500,550] \mathrm{Hz}$. Then the rank of the first acoustic mode in MF band $[500,550] \mathrm{Hz}$ is 129 and consequently, the acoustic cavity has an MF behavior in MF band $B$.

\section{Constructing the reference solution on a broad frequency band}

The reference solution is constructed on the $[5,700] \mathrm{Hz}$ broad frequency band with a sampling frequency step $\delta \nu=0.165 \mathrm{~Hz}$. The power spectral density functions $S_{\text {struc }}^{\text {ref }}(\omega)$ and $S_{\text {acous }}^{\text {ref }}(\omega)$ corresponding to the reference solution and defined by Eqs. (76) and (77) are calculated directly in the frequency domain, using Eqs. (80) and (81), without using the reduced model. Figures 11 and 12 show the mappings $\nu \mapsto 10 \times \log _{10}\left(S_{\text {struc }}^{\text {ref }}(2 \pi \nu)\right)$ and $\nu \mapsto$ $10 \times \log _{10}\left(S_{\text {acous }}^{\text {ref }}(2 \pi \nu)\right)$ respectively over the $[5,700] \mathrm{Hz}$ broad frequency band.

\section{Reference solution on the narrow MF band}

The reference solution on narrow MF band $B=[500,550] \mathrm{Hz}$ is constructed as explained in Section VIII C using the frequency resolution $\delta \nu=0.166 \mathrm{~Hz}$. The graphs of functions $\nu \mapsto 10 \times \log _{10}\left(S_{\text {struc }}^{\text {ref }}(2 \pi \nu)\right)$ and $\nu \mapsto 10 \times \log _{10}\left(S_{\text {acous }}^{\text {ref }}(2 \pi \nu)\right)$ (corresponding to Figures 11 and 12) of the reference solution on narrow MF band $[500,550] \mathrm{Hz}$ are used below to evaluate the accuracy of the response constructed using the reduced model (see the solid lines in Figures 14 to 17).

\section{E. Constructing the dominant eigensubspaces}

For the structure, we use the dominant eigenspace calculated in Section VII E. For the internal acoustic fluid, the dominant eigensubspace of the energy operator relative to band $B$ for the internal acoustic cavity with rigid walls (related to matrix $\left[E_{2}\right]$ defined by Eqs. (41) to (43)) is constructed using the method presented in Section V with $N_{2}=50$. Figure 13 shows the graph of the function $j \mapsto 10 \times \log _{10}\left(\lambda_{2}^{j}\right)$ for $j \in\{1,2, \ldots, 50\}$ in which $\lambda_{2}^{1}, \ldots, \lambda_{2}^{50}$ are the highest eigenvalues of the generalized symmetric eigenvalue problem defined by Eq. (36) for $a=2$. Figure 13 shows that the order $N_{2}$ of the reduced model is about 40 for band $B$.

\section{F. Reduced model adapted to the narrow MF band}

In this section, we present a comparison of the reference solution constructed in Section VIII $\mathrm{D}$ with the solution obtained by the reduced model constructed using the results of Sections IV and VI and Eqs. (76) and (77). As for the first example presented in Section VII, two reduced models are considered below. For the first reduced model, the parameters are $N_{1}^{S}=N_{1}=30$ (then $N_{0}=0$ ) and $N_{2}=45$. Figure 14 is related to the structure and shows the comparison between function $\nu \mapsto 10 \times \log _{10}\left(S_{\text {struc }}^{\text {ref }}(2 \pi \nu)\right)$ (solid line corresponding to the reference solution) and function $\nu \mapsto 10 \times \log _{10}\left(S_{\text {struc }}(2 \pi \nu)\right)$ (dashed line corresponding to the first reduced model) on narrow MF band $[500,550] \mathrm{Hz}$. Figure 15 is related to the internal acoustic fluid and shows the comparison between function $\nu \mapsto 10 \times \log _{10}\left(S_{\text {acous }}^{\text {ref }}(2 \pi \nu)\right)$ (solid line 
corresponding to the reference solution) and function $\nu \mapsto 10 \times \log _{10}\left(S_{\text {acous }}(2 \pi \nu)\right)$ (dashed line corresponding to the first reduced model) on narrow MF band $[500,550] \mathrm{Hz}$. Figures 14 and 15 show that the comparison is good for both the structure and the internal acoustic fluid.

For the second reduced model, the parameters are $N_{1}=30, N_{0}=10$ (then $N_{1}^{S}=40$ ) and $N_{2}=45$. Figure 16 is related to the structure and shows the comparison between function $\nu \mapsto 10 \times \log _{10}\left(S_{\text {struc }}^{\text {ref }}(2 \pi \nu)\right)$ (solid line corresponding to the reference solution) and function $\nu \mapsto 10 \times \log _{10}\left(S_{\text {struc }}(2 \pi \nu)\right.$ ) (dashed line corresponding to the second reduced model) on narrow MF band $[500,550] \mathrm{Hz}$. Figure 17 is related to the internal acoustic fluid and shows the comparison between function $\nu \mapsto 10 \times \log _{10}\left(S_{\text {acous }}^{\text {ref }}(2 \pi \nu)\right)$ (solid line corresponding to the reference solution) and function $\nu \mapsto 10 \times \log _{10}\left(S_{\text {acous }}(2 \pi \nu)\right)$ (dashed line corresponding to the second reduced model) on narrow MF band $[500,550] \mathrm{Hz}$. Figure 16 is quite similar to Figure 14 and Figure 17 to Figure 15. This means that for an MF-MF coupling, the reduced model of the structure can be constructed with $N_{0}=0$.

\section{CONCLUSIONS}

A theoretical approach is presented for constructing a reduced model in the MF range in the area of structural acoustics for a general three-dimensional anisotropic, inhomogeneous, viscoelastic bounded structure with an arbitrary geometry coupled with an internal acoustic fluid (gas or liquid). The boundary value problem formulated in the frequency domain and its variational formulation are presented. For a given MF band, the energy operator of the structure in vacuo and the energy operator of the internal acoustic cavity with rigid walls are positive-definite symmetric operators which have a countable set of decreasing positive eigenvalues. The eigenfunctions corresponding to the highest eigenvalues (dominant eigensubspace) of each energy operator constitute an appropriate functional basis of the corresponding admissible function space for the structure and for the internal acoustic fluid. For an MF structure (i.e. a structure having an MF behavior) coupled with an MF internal acoustic fluid (i.e. an internal acoustic fluid having an MF behavior) in the MF band considered, these two functional bases allow a reduced model of the structural-acoustic system to be constructed using the Ritz-Galerkin method. If the MF structure is coupled with an LF internal acoustic fluid, then it is more efficient to complete the structural vector basis relative to the MF band with a few LF structural modes in order to accelerate convergence of the solution for the internal acoustic cavity response in the MF band. A finite dimension approximation of the continuous case is introduced in a general context (using the finite element method or not). For construction of the dominant eigensubspace of each energy operator, an efficient procedure based on the use of the subspace iteration method is proposed. It does not require explicit calculation of the energy operator. We then obtain an efficient method for constructing a reduced model in the MF range: 1) an intrinsic reduced model is constructed for general structural-acoustic systems and can be considered as a progress independently of any computer time aspects; 2) the efficiency of such a reduced model in the MF range can be very high with respect to any other techniques validated in the MF range as soon as the MF responses have to be calculated for a large number of multiple deterministic and random loads, particularly for random excitations; 3) the implementation of this method is easily to peform in any usual finite element computer code. Finally, concerning the structure, the results presented can be extended straightforwardly to a structure made of beams, plates and shells. Analysis of the two examples (MF-LF coupling and MF-MF coupling) validates the reduced model methodology proposed for the MF range. 
1 C. Soize, "Reduced models in the medium-frequency range for general dissipative structuraldynamics systems," Eur. J. Mech. A/Solids 17(4),657-685 (1998).

2 C. Soize, "Reduced models in the medium-frequency range for general external structuralacoustic systems," J. Acoust. Soc. Am. 103(6), 3393-3406 (1998).

3 R. Ohayon and C. Soize, Structural Acoustics and Vibration (Academic Press, San Diego, 1998).

4 Y. C. Fung, Foundations of Solid Mechanics (Prentice Hall, Englewood Cliffs, New Jersey, 1968).

5 C. Truesdell, Mechanics of Solids, Vol. III, Theory of Viscoelasticity, Plasticity, Elastic Waves and Elastic Stability (Springer-Verlag, Berlin, 1984).

6 P. G. Ciarlet, Mathematical Elasticity, Vol.I: Three-Dimensional Elasticity (North-Holland, Amsterdam, 1988).

7 O. C. Zienkiewicz and R. L. Taylor, The Finite Element Method (McGraw-Hill, New York, 1989).

8 R. Dautray and J.-L. Lions, Mathematical Analysis and Numerical Methods for Science and Technology (Springer-Verlag, Berlin, 1992).

9 P. M. Morse and H. Feshbach, Methods of Theoretical Physics (McGraw-Hill, New York, 1953).

10 A. Leissa, Vibration of Plates and Vibration of Shells (Acoust. Soc. Am. Publications on Acoustics, Woodbury, 1993). Originally published by NASA, 1973.

11 A. D. Pierce, Acoustics: An Introduction to its Physical Principles and Applications (Acoust. Soc. Am. Publications on Acoustics, Woodbury, 1993). Originally published by McGrawHill, New York, 1981.

12 K. J. Bathe and E. L. Wilson, Numerical Methods in Finite Element Analysis ( Prentice Hall, New York, 1976).

13 B. N. Parlett, The Symmetric Eigenvalue Problem (Prentice Hall, Englewood Cliffs, New Jersey, 1980).

14 G. H. Golub and C. F. Van Loan, Matrix Computations, 2nd Edition (The John Hopkins University Press, Baltimore and London, 1989).

15 F. Chatelin, Eigenvalues of Matrices (Wiley, New York, 1993).

16 P. Kree and C. Soize, Mathematics of Random Phenomena (Reidel, Dordrecht, 1986).

17 C. Soize, The Fokker-Planck Equation for Stochastic Dynamical Systems and its Explicit Steady State Solutions (World Scientific, Singapore, 1994).

18 Y.K. Lin, Probabilistic Theory of Structural Dynamics (McGraw-Hill, New York, 1967). 


\section{LEGENDS ACCOMPANYING EACH FIGURE}

FIG. 1. Geometrical configuration of the structural-acoustic system.

FIG. 2. Rectangular thin plate with point masses, springs and dashpots, coupled with an acoustic cavity constituted of a bounded rectangular room filled with a gas (air).

FIG. 3. Graph of function $\nu \mapsto 10 \times \log _{10}\left(S_{\text {struc }}^{\text {ref }}(2 \pi \nu)\right)$ corresponding to the reference solution for the MF structure coupled with the LF internal acoustic fluid.

FIG. 4. Graph of function $\nu \mapsto 10 \times \log _{10}\left(S_{\text {acous }}^{\text {ref }}(2 \pi \nu)\right)$ corresponding to the reference solution for the LF internal acoustic fluid coupled with the MF structure.

FIG. 5. Graph of function $j \mapsto 10 \times \log _{10}\left(\lambda_{1}^{j}\right)$ showing the distribution of eigenvalues $\lambda_{1}^{j}$ of the energy operator of the MF structure in vacuo.

FIG. 6. Graph of function $j \mapsto 10 \times \log _{10}\left(\lambda_{2}^{j}\right)$ showing the distribution of eigenvalues $\lambda_{2}^{j}$ of the energy operator of the LF internal acoustic cavity with rigid walls.

FIG. 7. Reduced model of the dynamical response of the MF structure coupled with the LF internal acoustic fluid: comparison between function $\nu \mapsto 10 \times \log _{10}\left(S_{\text {struc }}^{\text {ref }}(2 \pi \nu)\right)$ (reference solution (solid line)) and function $\nu \mapsto 10 \times \log _{10}\left(S_{\text {struc }}(2 \pi \nu)\right)$ (reduced model for $N_{1}=25$, $N_{0}=0$ and $N_{2}=4$ (dashed line)).

FIG. 8. Reduced model of the dynamical response of the LF internal acoustic fluid coupled with the MF structure: comparison between function $\nu \mapsto 10 \times \log _{10}\left(S_{\text {acous }}^{\text {ref }}(2 \pi \nu)\right)$ (reference solution (solid line)) and function $\nu \mapsto 10 \times \log _{10}\left(S_{\text {acous }}(2 \pi \nu)\right)$ (reduced model for $N_{1}=25$, $N_{0}=0$ and $N_{2}=4$ (dashed line)).

FIG. 9. Reduced model of the dynamical response of the MF structure coupled with the LF internal acoustic fluid: comparison between function $\nu \mapsto 10 \times \log _{10}\left(S_{\text {struc }}^{\text {ref }}(2 \pi \nu)\right)$ (reference solution (solid line)) and function $\nu \mapsto 10 \times \log _{10}\left(S_{\text {struc }}(2 \pi \nu)\right)$ (reduced model for $N_{1}=25$, $N_{0}=10$ and $N_{2}=4$ (dashed line)).

FIG. 10. Reduced model of the dynamical response of the LF internal acoustic fluid coupled with the MF structure: comparison between function $\nu \mapsto 10 \times \log _{10}\left(S_{\text {acous }}^{\text {ref }}(2 \pi \nu)\right)$ (reference solution (solid line)) and function $\nu \mapsto 10 \times \log _{10}\left(S_{\text {acous }}(2 \pi \nu)\right)$ (reduced model for $N_{1}=25$, $N_{0}=10$ and $N_{2}=4$ (dashed line)).

FIG. 11. Graph of function $\nu \mapsto 10 \times \log _{10}\left(S_{\text {struc }}^{\text {ref }}(2 \pi \nu)\right)$ corresponding to the reference solution for the MF structure coupled with the MF internal acoustic fluid.

FIG. 12. Graph of function $\nu \mapsto 10 \times \log _{10}\left(S_{\text {acous }}^{\text {ref }}(2 \pi \nu)\right)$ corresponding to the reference solution for the MF internal acoustic fluid coupled with the MF structure.

FIG. 13. Graph of function $j \mapsto 10 \times \log _{10}\left(\lambda_{2}^{j}\right)$ showing the distribution of eigenvalues $\lambda_{2}^{j}$ of the energy operator of the MF internal acoustic cavity with rigid walls.

FIG. 14. Reduced model of the dynamical response of the MF structure coupled with the MF internal acoustic fluid: comparison between function $\nu \mapsto 10 \times \log _{10}\left(S_{\text {struc }}^{\text {ref }}(2 \pi \nu)\right)$ (reference solution (solid line)) and function $\nu \mapsto 10 \times \log _{10}\left(S_{\text {struc }}(2 \pi \nu)\right)$ (reduced model for $N_{1}=30$, $N_{0}=0$ and $N_{2}=45$ (dashed line)).

FIG. 15. Reduced model of the dynamical response of the MF internal acoustic fluid coupled with the MF structure: comparison between function $\nu \mapsto 10 \times \log _{10}\left(S_{\text {acous }}^{\text {ref }}(2 \pi \nu)\right)$ (reference solution (solid line)) and function $\nu \mapsto 10 \times \log _{10}\left(S_{\text {acous }}(2 \pi \nu)\right.$ ) (reduced model for $N_{1}=30$, $N_{0}=0$ and $N_{2}=45$ (dashed line)). 
FIG. 16. Reduced model of the dynamical response of the MF structure coupled with the MF internal acoustic fluid: comparison between function $\nu \mapsto 10 \times \log _{10}\left(S_{\text {struc }}^{\text {ref }}(2 \pi \nu)\right)$ (reference solution (solid line)) and function $\nu \mapsto 10 \times \log _{10}\left(S_{\text {struc }}(2 \pi \nu)\right)$ (reduced model for $N_{1}=30$, $N_{0}=10$ and $N_{2}=45$ (dashed line)).

FIG. 17. Reduced model of the dynamical response of the MF internal acoustic fluid coupled with the MF structure: comparison between function $\nu \mapsto 10 \times \log _{10}\left(S_{\text {acous }}^{\text {ref }}(2 \pi \nu)\right)$ (reference solution (solid line)) and function $\nu \mapsto 10 \times \log _{10}\left(S_{\text {acous }}(2 \pi \nu)\right)$ (reduced model for $N_{1}=30$, $N_{0}=10$ and $N_{2}=45$ (dashed line)). 


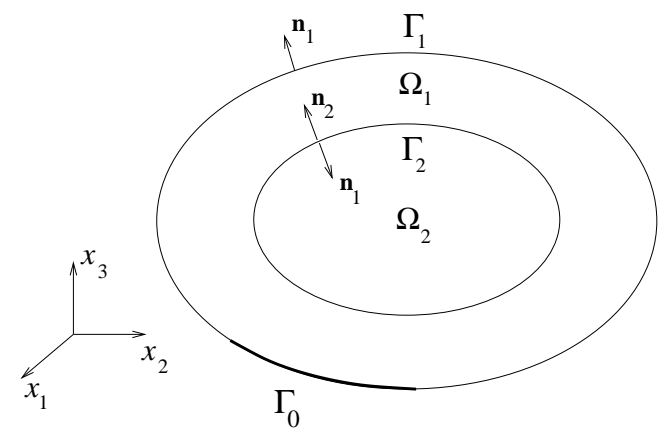

Fig. 1, Christian Soize, J. Acoust. Soc. Am.

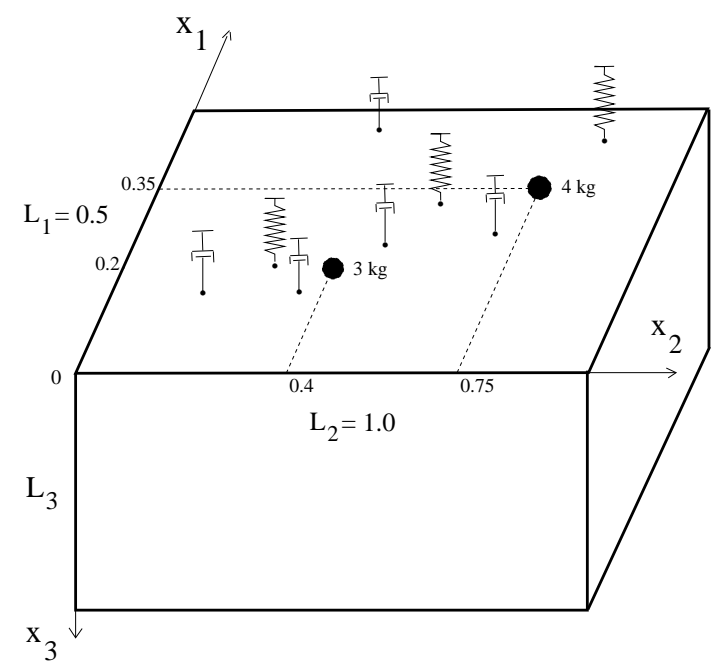

Fig. 2, Christian Soize, J. Acoust. Soc. Am.

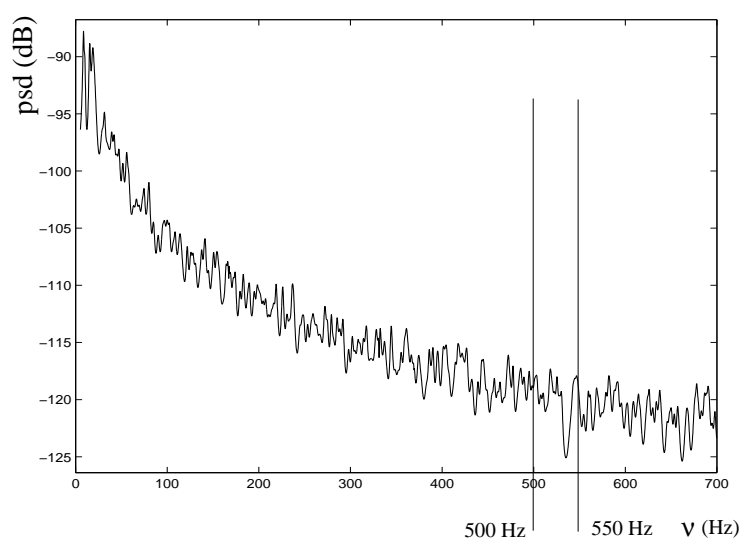

Fig. 3, Christian Soize, J. Acoust. Soc. Am.

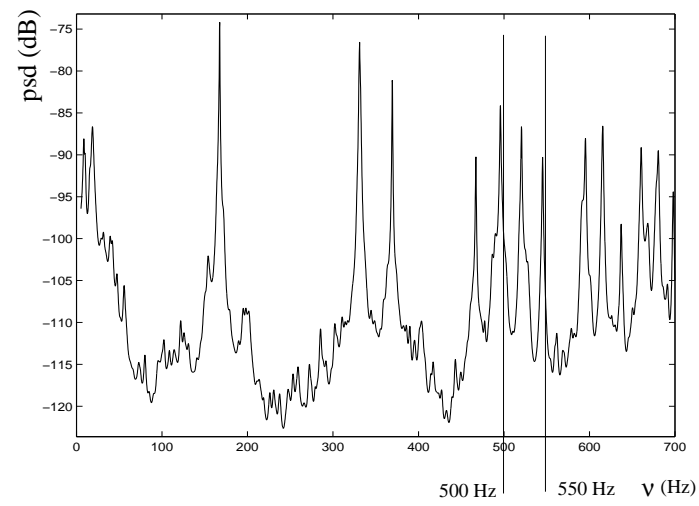

Fig. 4, Christian Soize, J. Acoust. Soc. Am.

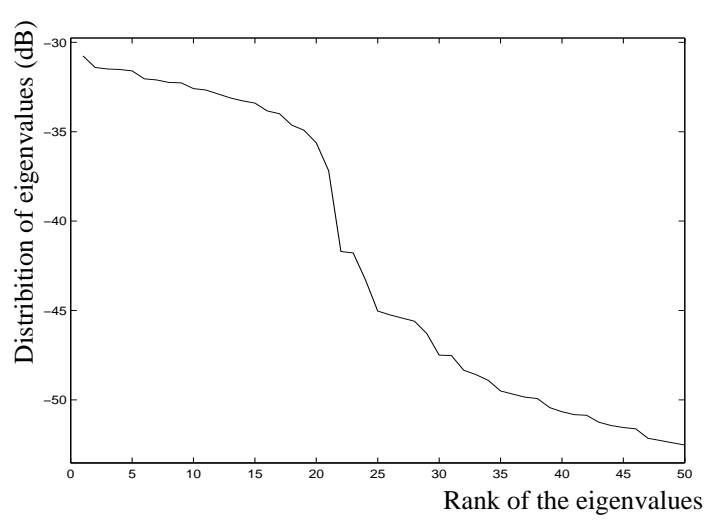

Fig. 5, Christian Soize, J. Acoust. Soc. Am.

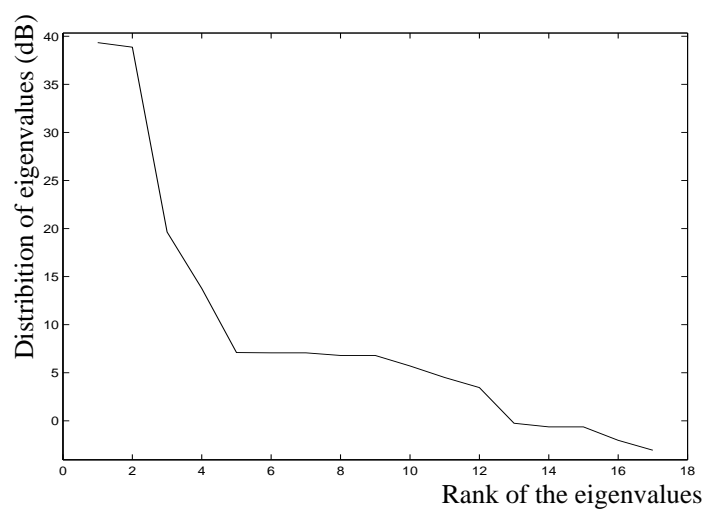

Fig. 6, Christian Soize, J. Acoust. Soc. Am. 


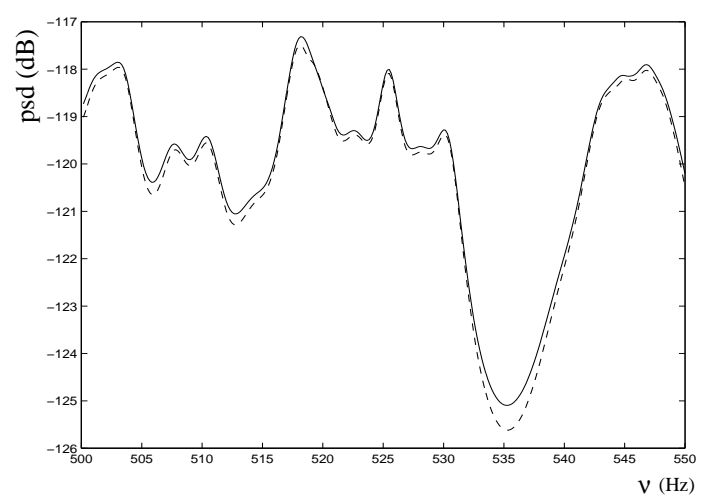

Fig. 7, Christian Soize, J. Acoust. Soc. Am.

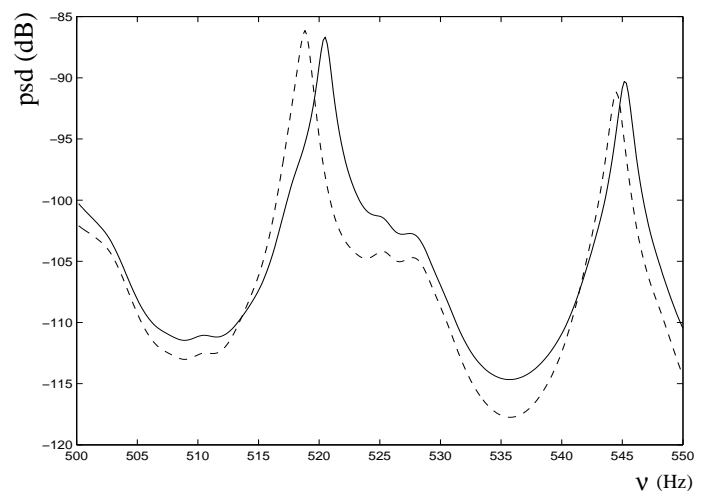

Fig. 8, Christian Soize, J. Acoust. Soc. Am.

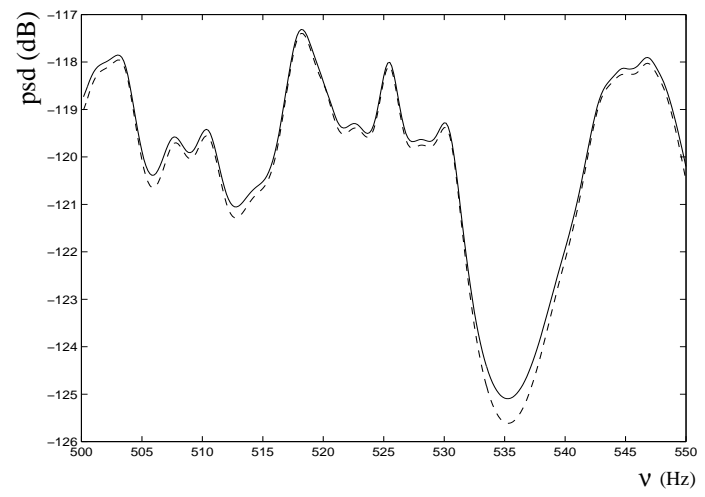

Fig. 9, Christian Soize, J. Acoust. Soc. Am.

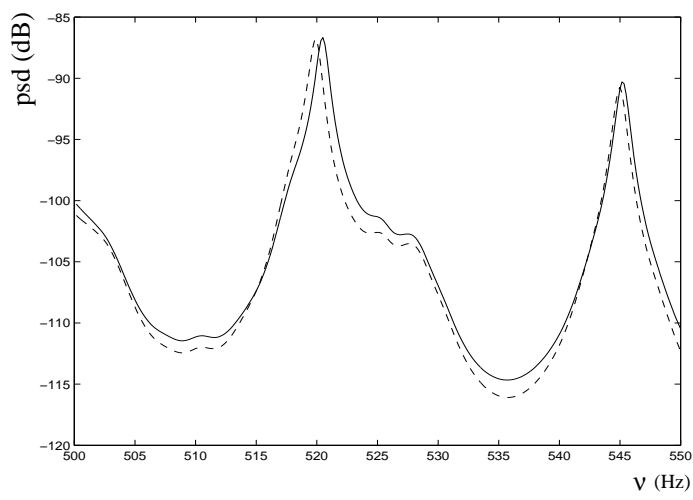

Fig. 10, Christian Soize, J. Acoust. Soc. Am.

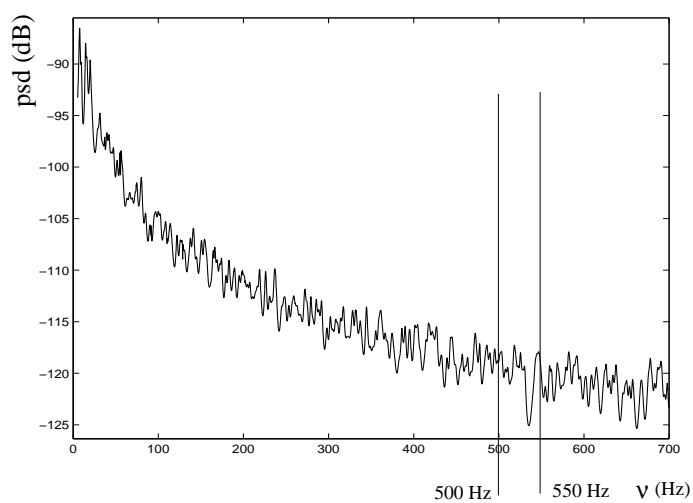

Fig. 11, Christian Soize, J. Acoust. Soc. Am.

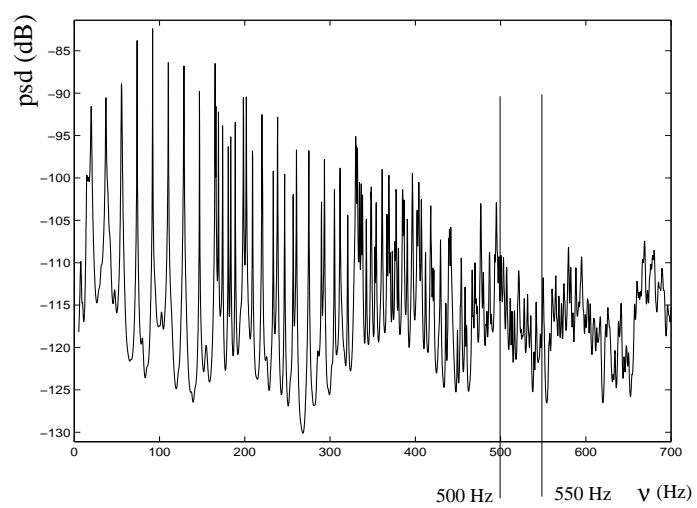

Fig. 12, Christian Soize, J. Acoust. Soc. Am. 


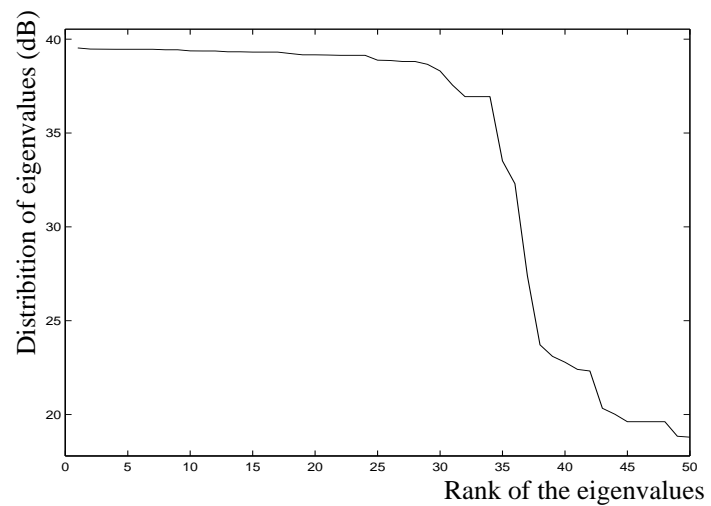

Fig. 13, Christian Soize, J. Acoust. Soc. Am.

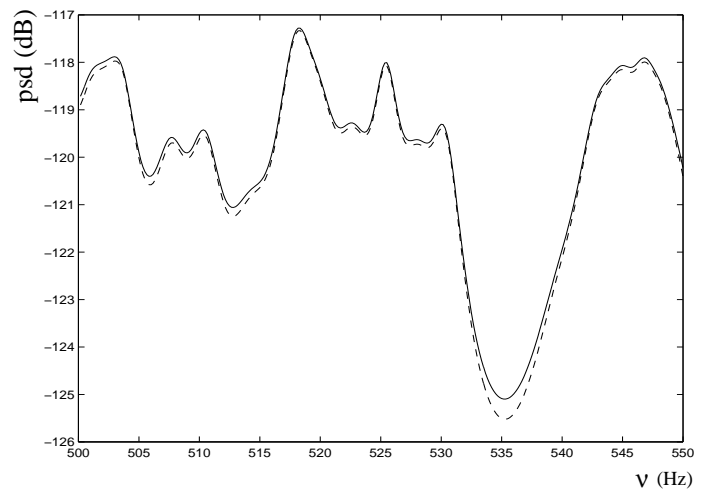

Fig. 14, Christian Soize, J. Acoust. Soc. Am.

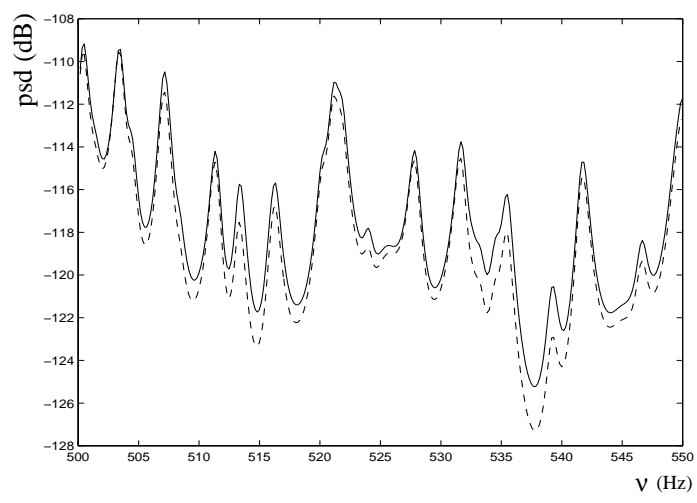

Fig. 15, Christian Soize, J. Acoust. Soc. Am.

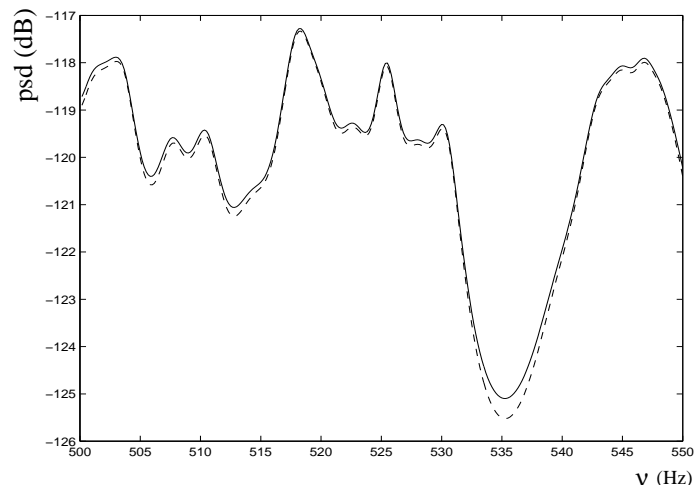

Fig. 16, Christian Soize, J. Acoust. Soc. Am.

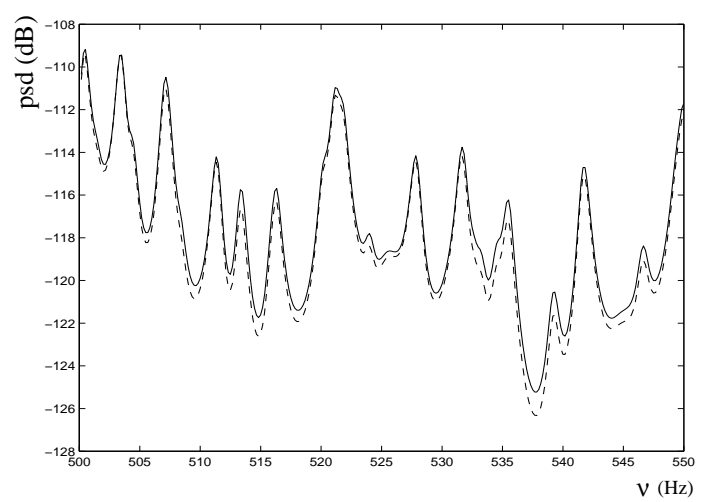

Fig. 17, Christian Soize, J. Acoust. Soc. Am. 